\title{
THE COMPLEXITY OF LINEAR-TIME TEMPORAL LOGIC OVER THE CLASS OF ORDINALS*
}

\author{
STÉPHANE DEMRI $^{a}$ AND ALEXANDER RABINOVICH ${ }^{b}$
}

${ }^{a}$ LSV, ENS Cachan, CNRS, INRIA Saclay IdF, 61, av. Pdt. Wilson, 94235 Cachan Cedex, France

e-mail address: demri@lsv.ens-cachan.fr

${ }^{b}$ School of Computer Science, Tel Aviv University, Ramat Aviv, Tel Aviv 69978, Israel

e-mail address: rabinoa@post.tau.ac.il

\begin{abstract}
We consider the temporal logic with since and until modalities. This temporal logic is expressively equivalent over the class of ordinals to first-order logic by Kamp's theorem. We show that it has a PSPACE-complete satisfiability problem over the class of ordinals. Among the consequences of our proof, we show that given the code of some countable ordinal $\alpha$ and a formula, we can decide in PSPACE whether the formula has a model over $\alpha$. In order to show these results, we introduce a class of simple ordinal automata, as expressive as Büchi ordinal automata. The PSPACE upper bound for the satisfiability problem of the temporal logic is obtained through a reduction to the nonemptiness problem for the simple ordinal automata.
\end{abstract}

\section{INTRODUCTION}

The main models for time are $\langle\mathbb{N},<\rangle$, the natural numbers as a model of discrete time and the structure $\langle\mathbb{R},<\rangle$, the real line as the model for continuous time. These two models are called the canonical models of time. A major result concerning linear-time temporal logics is Kamp theorem [Kam68, GHR94 which says that LTL(U,S), the temporal logic having "Until" and "Since" as only modalities, is expressively complete for first-order monadic logic of order over the class of Dedekind-complete linear orders. The canonical models of time are indeed Dedekind-complete. Another important class of Dedekind-complete orders is the class of ordinals.

In this paper, the satisfiability problem for the temporal logic with until and since modalities over the class of ordinals is investigated. This is the opportunity to generalize what is known about the logic over $\omega$-sequences. Our main results are the following.

(1) The satisfiability problem for $\operatorname{LTL}(\mathrm{U}, \mathrm{S})$ over the class of ordinals is PSPACE-complete.

1998 ACM Subject Classification: F.4.1, F.3.1., F.2.2.

Key words and phrases: linear-time temporal logic, ordinal, polynomial space, automaton.

* The extended abstract of this paper appeared in DR07.

${ }^{a}$ Partially supported by project AutoMathA (ESF).

${ }^{b}$ Partially supported by an invited professorship from ENS de Cachan. 
(2) A formula $\phi$ in $\operatorname{LTL}(\mathrm{U}, \mathrm{S})$ has some $\alpha$-model for some ordinal $\alpha$ iff it has an $\beta$-model for some $\beta<\omega^{|\phi|+2}$ where $|\phi|$ denotes the size of $\phi$ for some reasonably succinct encoding (see forthcoming Corollary 3.3).

In order to prove these results we use an automata-based approach [Büc62, VW94]. In Section 2, we introduce a new class of ordinal automata which we call simple ordinal automata. These automata are expressive equivalent to Büchi automata over countable ordinals [BS73]. However, the locations and the transition relations of these automata have additional structures as in [Roh97]. In particular, a location is a subset of a base set $B$. Herein, we provide a translation from formulae in $\operatorname{LTL}(\mathrm{U}, \mathrm{S})$ into simple ordinal automata that allows to characterize the complexity of the satisfiability problem for LTL(U,S). However, the translation of the formula $\phi$ into the automaton $\mathcal{A}_{\phi}$ provides an automaton of exponential size in $|\phi|$ but the cardinal of the basis of $\mathcal{A}_{\phi}$ is linear in $|\phi|$.

Section 3 contains our main technical lemmas. We show there that every run in a simple ordinal automaton is equivalent to a short run. Consequently, we establish that a formula $\phi \in \operatorname{LTL}(\mathrm{U}, \mathrm{S})$ has an $\alpha$-model for some countable ordinal $\alpha$ iff it has a model of length $\operatorname{trunc}_{|\phi|+2}(\alpha)$ where trunc $\left.\right|_{|\phi|+2}(\alpha)$ is a truncated part of $\alpha$ strictly less than $\omega^{|\phi|+2} \times 2$ (see the definition of truncation in Section 3). In Section 4 we present two algorithms to solve the nonemptiness problem for simple ordinal automata. The first one runs in (simple) exponential time and does not take advantage of the short run property. The second algorithm runs in polynomial space and the short run property plays the main role in its design and its correctness proof.

In Section 5 we investigate several variants of the satisfiability problem and show that all of them are PSPACE-complete. Section 6 compares our results with related works. The satisfiability problem for LTL(U,S) over $\omega$-models is PSPACE-complete [SC85]. Reynolds Rey03, Rey10b proved that the satisfiability problem for LTL(U,S) over the reals is PSPACEcomplete. The proofs in Rey03, Rey10b are non trivial and difficult to grasp and it is therefore difficult to compare our proof technique with those of [Rey03, Rey10b] even though we believe cross-fertilization would be fruitful. We provide uniform proofs and we improve upper bounds for decision problems considered in [Cac06, DN07, Roh97, see also [BLW07. We also compare our results and techniques with Rohde's thesis [Roh97]. Finally we show how our results entail most of the results from [DN07] and we solve some open problems stated there.

\section{Linear-Time Temporal Logic with Until and Since}

1.1. Basic definitions on ordinals. Let us start smoothly by recalling basic definitions and properties about ordinals, see e.g. [Ros82] for additional material. An ordinal is a totally ordered set which is well ordered, i.e. all its non-empty subsets have a least element. Order-isomorphic ordinals are considered equal. They can be more conveniently defined inductively by: the empty set (written 0 ) is an ordinal, if $\alpha$ is an ordinal, then $\alpha \cup\{\alpha\}$ (written $\alpha+1$ ) is an ordinal and, if $X$ is a set of ordinals, then $\bigcup_{\alpha \in X} \alpha$ is an ordinal. The ordering is obtained by $\beta<\alpha$ iff $\beta \in \alpha$. An ordinal $\alpha$ is a successor ordinal iff there exists an ordinal $\beta$ such that $\alpha=\beta+1$. An ordinal which is not 0 or a successor ordinal, is a limit ordinal. The first limit ordinal is written $\omega$. Addition, multiplication and exponentiation can be defined on ordinals inductively: $\alpha+0=\alpha, \alpha+(\beta+1)=(\alpha+\beta)+1$ and $\alpha+\beta=\sup \{\alpha+\gamma: \gamma<\beta\}$ where $\beta$ is a limit ordinal. Multiplication and exponentiation 
are defined similarly. Whenever $\alpha \leq \beta$, there is a unique ordinal $\gamma$ such that $\alpha+\gamma=\beta$ and we write $\beta-\alpha$ to denote $\gamma$.

1.2. Temporal logic. The formulae of $\operatorname{LTL}(U, S)$ are defined as follows:

$$
\phi::=p|\neg \phi| \phi_{1} \wedge \phi_{2}\left|\phi_{1} \mathrm{U}_{2}\right| \phi_{1} \mathrm{~S} \phi_{2}
$$

where $p \in$ PROP for some countably infinite set PROP of atomic propositions. Given a formula $\phi$ in $\operatorname{LTL}(\mathrm{U}, \mathrm{S})$, we write $\operatorname{sub}(\phi)$ to denote the set of subformulae of $\phi$ or their negation assuming that $\neg \neg \psi$ is identified with $\psi$. The size of $\phi$ is defined as the cardinality of $\operatorname{sub}(\phi)$ and therefore implicitly we encode formulae as DAGs, which is exponentially more succinct that the representation by trees. This feature will be helpful for defining translations that increase only polynomially the number of subformulae but for which the tree representation might suffer an exponential blow-up. We use the following standard abbreviations $\mathrm{G} \phi=\phi \wedge \neg(\top \mathrm{U} \neg \phi), \mathrm{G}^{+} \phi=\neg(\mathrm{TU} \neg \phi), \mathrm{F} \phi=\neg \mathrm{G} \neg \phi, \mathrm{F}^{+} \phi=\neg \mathrm{G}^{+} \neg \phi, \mathrm{X} \phi=\perp$ $\mathrm{U} \phi$ and $\mathrm{X}^{-1} \phi=\perp \mathrm{S} \phi$ that do cause only a polynomial increase in size.

An $\alpha$-model $\sigma$ is a function $\sigma: \alpha \rightarrow \mathcal{P}(\mathrm{PROP})$ for some ordinal $\alpha \neq 0$. The satisfaction relation " $\phi$ holds in the $\alpha$-model $\sigma$ at position $\beta$ " $(\beta<\alpha)$ is defined as follows:

- $\sigma, \beta \models p$ iff $p \in \sigma(\beta)$,

- $\sigma, \beta \models \neg \phi$ iff not $\sigma, \beta \models \phi$,

- $\sigma, \beta \models \phi_{1} \wedge \phi_{2}$ iff $\sigma, \beta \models \phi_{1}$ and $\sigma, \beta \models \phi_{2}$,

- $\sigma, \beta \models \phi_{1} \mathrm{U} \phi_{2}$ iff there is $\gamma \in(\beta, \alpha)$ such that $\sigma, \gamma \models \phi_{2}$ and for every $\gamma^{\prime} \in(\beta, \gamma)$, we have $\sigma, \gamma^{\prime} \models \phi_{1}$,

- $\sigma, \beta \models \phi_{1} \mathrm{~S} \phi_{2}$ iff there is $\gamma \in[0, \beta)$ such that $\sigma, \gamma \models \phi_{2}$ and for every $\gamma^{\prime} \in(\gamma, \beta)$, we have $\sigma, \gamma^{\prime} \models \phi_{1}$.

Observe that S and U are strict "since" and "until" modalities.

The (initial) satisfiability problem for LTL(U,S) consists in determining, given a formula $\phi$, whether there is a model $\sigma$ such that $\sigma, 0 \models \phi$. Note that $\phi$ is satisfiable in a model $\sigma$ iff $\mathrm{F} \phi$ is initially satisfiable in $\sigma$. Therefore, there is a polynomial-time reduction from the satisfiability problem to the initial satisfiability problem. From now on, we will deal only with the initial satisfiability problem and for the sake of brevity we will call it "satisfiability problem".

We recall that well orders are particular cases of Dedekind complete linear orders. Indeed, a chain is Dedekind complete iff every non-empty bounded subset has a least upper bound. Kamp's theorem applies herein.

Theorem 1.1. Kam68] LTL(U,S) over the class of ordinals is as expressive as the firstorder logic.

Moreover, satisfiability for $\operatorname{LTL}(\mathrm{U}, \mathrm{S})$ is known to be decidable and as stated below we can restrict ourselves to countable models.

\section{Theorem 1.2.}

(I): BS73] The satisfiability problem for $\operatorname{LTL}(\mathrm{U}, \mathrm{S})$ over the class of countable ordinals is decidable.

(II): (see e.g. GS85, Lemma 6]) A formula in $\operatorname{LTL}(\mathrm{U}, \mathrm{S})$ is satisfiable iff it is satisfiable in a model of length some countable ordinal. 
Observe that in BS73] it was proved that monadic second-order logic over the class of countable ordinals is decidable and in [GS85] it was shown that if a formula of the first-order monadic logic is satisfiable in a model over an ordinal then it is satisfiable in a model over a countable ordinal. (I) and (II) are immediate consequences of these results and the fact that $\operatorname{LTL}(\mathrm{U}, \mathrm{S})$ can be easily translated into first-order logic.

Consequently, LTL $(\mathrm{U}, \mathrm{S})$ over the class of ordinals is certainly a fundamental logic to be studied. We recall below a central complexity result that we will extend to all ordinals.

Theorem 1.3. SC85] Satisfiability for $\operatorname{LTL}(\mathrm{U}, \mathrm{S})$, restricted to $\omega$-models, is PSPACE- complete.

\section{TRANSLATion From formulae to Simple ordinal AUtOMATA}

In Section 2.1, we introduce a new class of ordinal automata which we call simple ordinal automata. These automata are expressive equivalent to Büchi automata over ordinals [BS73]. However, the locations and the transition relations of these automata have additional structures. In Section 2.3, we provide a translation from $\operatorname{LTL}(\mathrm{U}, \mathrm{S})$ into simple ordinal automata which assigns to every formula in $\operatorname{LTL}(\mathrm{U}, \mathrm{S})$ an automaton that recognizes exactly its models. We borrow the automata-based approach for temporal logics from [VW94, KVW00].

\subsection{Simple ordinal automata.}

Definition 2.1. A simple ordinal automaton $\mathcal{A}$ is a structure $\left\langle B, Q, \delta_{\text {next }}, \delta_{\text {lim }}\right\rangle$ such that

- $B$ is a finite set (the basis of $\mathcal{A}$ ),

- $Q \subseteq \mathcal{P}(B)$ (the set of locations),

- $\delta_{\text {next }} \subseteq Q \times Q$ is the next-step transition relation,

- $\delta_{\text {lim }} \subseteq \mathcal{P}(B) \times Q$ is the limit transition relation.

$\mathcal{A}$ can be viewed as a finite directed graph whose set of nodes is structured. Limit transitions, whose interpretation is given below, allow reaching a node after an infinite amount of steps. Given a simple ordinal automaton $\mathcal{A}$, an $\alpha$-path (or simply a path) is a map $r: \alpha \rightarrow Q$ for some $\alpha>0$ such that

- for every $\beta+1<\alpha,\langle r(\beta), r(\beta+1)\rangle \in \delta_{\text {next }}$,

- for every limit ordinal $\beta<\alpha,\left\langle B_{\text {lim }}(r, \beta), r(\beta)\right\rangle \in \delta_{\text {lim }}$ where

$$
B_{\text {lim }}(r, \beta) \stackrel{\text { def }}{=}\left\{a \in B: \exists \gamma<\beta \text { such that for every } \gamma^{\prime} \in(\gamma, \beta), a \in r\left(\gamma^{\prime}\right)\right\} .
$$

The set $B_{\text {lim }}(r, \beta)$ contains exactly the elements of the basis that belong to every location from some $\gamma<\beta$ until $\beta$. We sometimes write $B_{l i m}(r)$ instead of $B_{l i m}(r, \alpha)$ when $\alpha$ is a limit ordinal.

Given an $\alpha$-path $r$, for $\beta, \beta^{\prime}<\alpha$ we write

- $r_{\geq \beta}$ to denote the restriction of $r$ to positions greater or equal to $\beta$,

- $r_{\leq \beta}$ to denote the restriction of $r$ to positions less or equal to $\beta$,

- $r_{\left[\beta, \beta^{\prime}\right)}$ to denote the restriction of $r$ to positions in $\left[\beta, \beta^{\prime}\right.$ ) (half-open interval).

A simple ordinal automaton with acceptance conditions is a structure of the form

$$
\left\langle B, Q, I, F, \mathcal{F}, \delta_{\text {next }}, \delta_{\text {lim }}\right\rangle
$$

where 
- $I \subseteq Q$ is the set of initial locations,

- $F \subseteq Q$ is the set of final locations for accepting runs whose length is some successor ordinal,

- $\mathcal{F} \subseteq \mathcal{P}(B)$ encodes the accepting condition for runs whose length is some limit ordinal.

Given a simple ordinal automaton with acceptance conditions, an accepting run is a path $r: \alpha \rightarrow Q$ such that

- $r(0) \in I$,

- if $\alpha$ is a successor ordinal, then $r(\alpha-1) \in F$, otherwise $B_{\text {lim }}(r) \in \mathcal{F}$.

The nonemptiness problem for simple ordinal automata consists in checking whether $\mathcal{A}$ has an accepting run. Our current definition for simple ordinal automata does not make them language acceptors since they have no alphabet. It is possible to add in the definition a finite alphabet $\Sigma$ and to define the next-step transition relation as a subset of $Q \times \Sigma \times Q$, see an example on the right-hand side of Figure 1, Additionally, the current definition can be viewed as the case either when the alphabet is a singleton or when the read letter is encoded in the locations through the dedicated elements of the basis. This second reading will be in fact used implicitly in the sequel. We also write $\mathcal{A}$ to denote either a simple ordinal automaton or its extension with acceptance conditions.

2.2. Relationships with Büchi automata. Simple ordinal automata with acceptance conditions and alphabet define the same class of languages as standard ordinal automata in the sense of [Büc64, Büc65]. Main arguments are provided below for the sake of completeness. However, we do not need this correspondence in our forthcoming developments. The main interest for our model of simple ordinal automata rests on the fact that it allows us to obtain the promised PSPACE upper bound. A standard ordinal automaton is a structure $\mathcal{A}=\left\langle\Sigma, Q, I, F, \mathcal{F}, \delta_{\text {next }}, \delta_{\text {lim }}\right\rangle$ such that

- $\Sigma$ is a finite alphabet,

- $Q$ is a finite set of locations,

- $\delta_{\text {next }} \subseteq Q \times \Sigma \times Q$ and $\delta_{\text {lim }} \subseteq \mathcal{P}(Q) \times Q$,

- $I, F \subseteq Q$ and $\mathcal{F} \subseteq \mathcal{P}(Q)$.

A word $u: \alpha \rightarrow \Sigma$ is accepted by $\mathcal{A}$ iff there is $r: \alpha \rightarrow Q$ such that

- for every $\beta+1<\alpha,\langle r(\beta), u(\beta), r(\beta+1)\rangle \in \delta_{\text {next }}$,

- for every limit ordinal $\beta<\alpha$, $\langle\inf (r, \beta), r(\beta)\rangle \in \delta_{\text {lim }}$ where

$$
\inf (r, \beta) \stackrel{\text { def }}{=}\left\{q \in Q: \text { for all } \gamma<\beta \text { there is } \gamma^{\prime} \in(\alpha, \beta) \text { such that } r\left(\gamma^{\prime}\right)=q\right\} .
$$

As usual, $\inf (r, \beta)$ denotes the set of locations that appear cofinally before $\beta$.

- $r(0) \in I$ and if $\alpha$ is a successor ordinal, then $r(\alpha-1) \in F$, otherwise $\inf (r, \alpha) \in \mathcal{F}$.

We write $\mathrm{L}(\mathcal{A})$ to denote the set of words accepted by $\mathcal{A}$. Similar definitions can be given for simple ordinal automata with acceptance conditions and alphabet.

Lemma 2.2.

(I): Given a simple ordinal automaton $\mathcal{A}$, there is a standard ordinal automaton $\mathcal{A}^{\prime}$ such that $\mathrm{L}(\mathcal{A})=\mathrm{L}\left(\mathcal{A}^{\prime}\right)$.

(II): Given a standard ordinal automaton $\mathcal{A}$, there is a simple ordinal automaton $\mathcal{A}^{\prime}$ such that $\mathrm{L}(\mathcal{A})=\mathrm{L}\left(\mathcal{A}^{\prime}\right)$. 


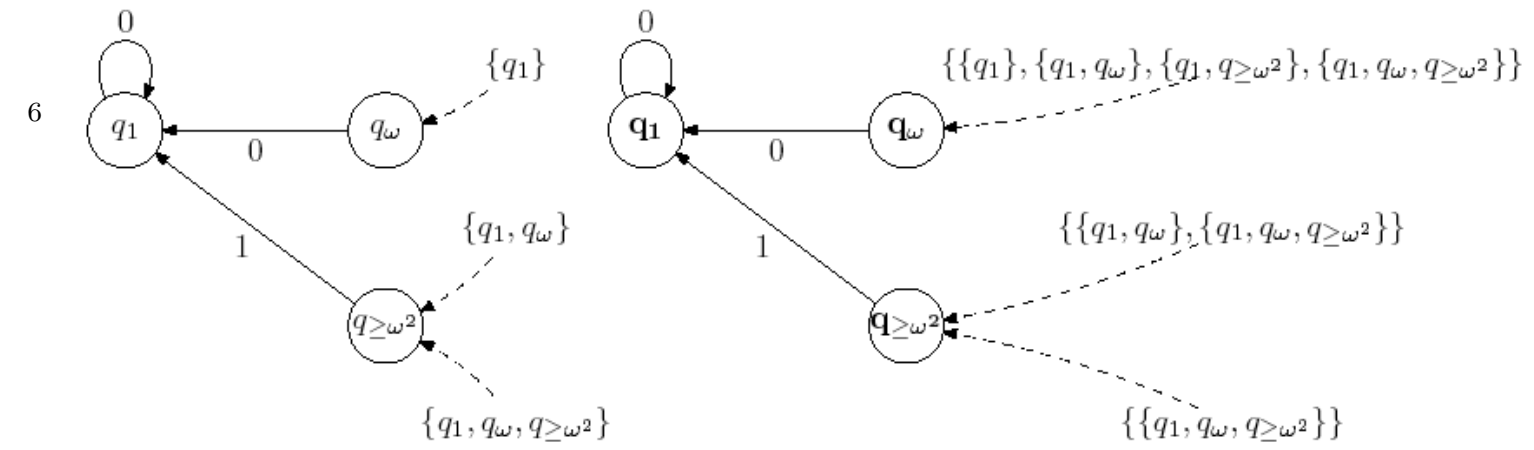

Figure 1: Ordinal automata accepting $\mathrm{L}_{0}$

Proof. (I) Let $\mathcal{A}$ be a simple ordinal automaton $\mathcal{A}=\left\langle\Sigma, B, Q, I, F, \mathcal{F}, \delta_{\text {next }}, \delta_{\text {lim }}\right\rangle$. We consider the standard ordinal automaton $\mathcal{A}^{\prime}$ of the form $\left\langle\Sigma, Q, I, F, \mathcal{F}, \delta_{\text {next }}, \delta_{\text {lim }}^{\prime}\right\rangle$ such that $\langle Y, q\rangle \in \delta_{\text {lim }}^{\prime}$ iff there is a limit transition $\langle Z, q\rangle \in \delta_{\text {lim }}$ satisfying the conditions below.

- for every $q^{\prime} \in Y$, we have $Z \subseteq q^{\prime}$,

- for every element $a \in(B \backslash Z)$, there is $q^{\prime} \in Y$ such that $a \notin q^{\prime}$.

One can easily check that $\mathrm{L}(\mathcal{A})=\mathrm{L}\left(\mathcal{A}^{\prime}\right)$. Observe that $\mathcal{A}^{\prime}$ can be exponentially larger than $\mathcal{A}$.

(II) Let $\mathcal{A}=\left\langle\Sigma, Q, I, F, \mathcal{F}, \delta_{\text {next }}, \delta_{\text {lim }}\right\rangle$ be a standard ordinal automaton. We build a simple ordinal automaton $\mathcal{A}^{\prime}=\left\langle\Sigma, B^{\prime}, Q^{\prime}, I^{\prime}, F^{\prime}, \mathcal{F}^{\prime}, \delta_{\text {next }}^{\prime}, \delta_{\text {lim }}^{\prime}\right\rangle$ as follows.

- $B^{\prime}=\mathcal{P}(Q)$.

- $Q^{\prime}=\left\{X \in \mathcal{P}\left(B^{\prime}\right): \exists q \in Q, X=\{Y \in \mathcal{P}(Q): q \in Y\}\right\}$. Below, when $q \in Q$, by abusing notation, we also write $q$ to denote the corresponding location in $Q^{\prime}$ equal to $\{Y \in \mathcal{P}(Q): q \in Y\}$.

- $I^{\prime}=I, F^{\prime}=F$ and $\mathcal{F}^{\prime}=\mathcal{F}$.

- For $a \in \Sigma$ and $q, q^{\prime} \in Q,\left\langle q, a, q^{\prime}\right\rangle \in \delta_{\text {next }}^{\prime}$ only if $\left\langle q, a, q^{\prime}\right\rangle \in \delta_{\text {next }}$.

- For $Y^{\prime} \subseteq B^{\prime}$ and $q \in Q,\left\langle Y^{\prime}, q\right\rangle \in \delta_{\text {lim }}^{\prime}$ only if there is a limit transition $\langle Y, q\rangle \in \delta_{\text {lim }}$ such that $Y^{\prime}=\left\{a \in B^{\prime}: Y \subseteq a\right\}$.

Again, one can easily check that $\mathrm{L}(\mathcal{A})=\mathrm{L}\left(\mathcal{A}^{\prime}\right)$.

Let $\mathrm{L}_{0}$ be the set of words $u: \alpha \rightarrow\{0,1\}$ such that for $\beta<\alpha, \beta=\omega^{2} \gamma$ for some ordinal $\gamma$ iff $u(\beta)=1$. The left-hand side of Figure 1 presents a standard ordinal automaton (with three locations) accepting $\mathrm{L}_{0}$. Next-step transitions are represented by plain arrows whereas limit transitions are represented by dashed arrows. Moreover, $F=\left\{q_{1}, q_{\omega}, q_{\geq \omega^{2}}\right\}$ and $\mathcal{F}=\mathcal{P}\left(\left\{q_{1}, q_{\omega}, q_{\geq \omega^{2}}\right\}\right)$. The right-hand side of Figure 1 presents a corresponding simple ordinal automaton along the lines of the proof of Lemma 2.2. Its basis $B$ is equal to $\mathcal{P}\left(\left\{q_{1}, q_{\omega}, q_{\geq \omega^{2}}\right\}\right)$ and we write $\mathbf{q}_{\mathbf{1}}$ to denote $\left\{\left\{q_{1}\right\},\left\{q_{1}, q_{\omega}\right\},\left\{q_{1}, q_{\geq \omega^{2}}\right\},\left\{q_{1}, q_{\omega}, q_{\geq \omega^{2}}\right\}\right\}$. $\mathbf{q}_{\omega}$ and $\mathbf{q}_{\geq \omega^{2}}$ are defined similarly.

2.3. Translation from $\operatorname{LTL}(\mathrm{U}, \mathrm{S})$ formulae to simple ordinal automata. As usual, a set $Y$ is a maximally Boolean consistent subset of $s u b(\phi)$ when the following conditions are satisfied:

- for every $\psi \in \operatorname{sub}(\phi), \neg \psi \in Y$ iff $\psi \notin Y$,

- for every $\psi_{1} \wedge \psi_{2} \in \operatorname{sub}(\phi), \psi_{1} \wedge \psi_{2} \in Y$ iff $\psi_{1}, \psi_{2} \in Y$.

Given a formula $\phi$, the simple ordinal automaton $\mathcal{A}_{\phi}=\left\langle B, Q, I, F, \mathcal{F}, \delta_{\text {next }}, \delta_{\text {lim }}\right\rangle$ is defined as follows:

- $B=\operatorname{sub}(\phi)$.

- $Q$ is the set of maximally Boolean consistent subsets of $s u b(\phi)$.

- $I$ is the set of locations that contain $\phi$ and no elements of the form $\psi_{1} S \psi_{2}$. 
- $F$ is the set of locations with no elements of the form $\psi_{1} \mathrm{U} \psi_{2}$.

- A subset $Y$ of $B$ is in $\mathcal{F}$ if there are no $\psi_{1}$ and $\psi_{2}$ such that $\left\{\psi_{1}, \neg \psi_{2}, \psi_{1} \mathrm{U} \psi_{2}\right\} \subseteq Y$.

- For all $q, q^{\prime} \in Q,\left\langle q, q^{\prime}\right\rangle \in \delta_{\text {next }} \stackrel{\text { def }}{\Leftrightarrow}$ the conditions below are satisfied:

$\left(\right.$ next $\left._{\mathrm{U}}\right)$ : for every $\psi_{1} \mathrm{U} \psi_{2} \in \operatorname{sub}(\phi), \psi_{1} \mathrm{U} \psi_{2} \in q$ iff either $\psi_{2} \in q^{\prime}$ or $\psi_{1}, \psi_{1} \mathrm{U} \psi_{2} \in q^{\prime}$, (nexts): for every $\psi_{1} \mathrm{~S} \psi_{2} \in \operatorname{sub}(\phi), \psi_{1} \mathrm{~S} \psi_{2} \in q^{\prime}$ iff either $\psi_{2} \in q$ or $\psi_{1}, \psi_{1} \mathrm{~S} \psi_{2} \in q$.

- For all $Y \subseteq B$ and $q \in Q,\langle Y, q\rangle \in \delta_{\text {lim }} \stackrel{\text { def }}{\Leftrightarrow}$ the conditions below are satisfied:

$\left(\lim _{\mathrm{U}} 1\right)$ : if $\psi_{1}, \neg \psi_{2}, \psi_{1} \mathrm{U} \psi_{2} \in Y$, then either $\psi_{2} \in q$ or $\psi_{1}, \psi_{1} \mathrm{U} \psi_{2} \in q$,

$\left(\lim _{\cup} 2\right)$ : if $\psi_{1}, \psi_{1} \mathrm{U} \psi_{2} \in q$ and $\psi_{1} \in Y$, then $\psi_{1} \mathrm{U} \psi_{2} \in Y$,

$\left(\lim _{\mathrm{U}} 3\right)$ : if $\psi_{1} \in Y, \psi_{2} \in q$ and $\psi_{1} \cup \psi_{2}$ is in the basis $B$, then $\psi_{1} \cup \psi_{2} \in Y$,

$\left(\lim _{\mathrm{S}}\right)$ : for every $\psi_{1} \mathrm{~S} \psi_{2} \in \operatorname{sub}(\phi), \psi_{1} \mathrm{~S} \psi_{2} \in q$ iff $\left(\psi_{1} \in Y\right.$ and $\left.\psi_{1} \mathrm{~S} \psi_{2} \in Y\right)$.

Even though the conditions above are compatible with the intuition that a location contains the formulae that are promised to be satisfied, at the current stage it might sound mysterious how the conditions have been made up (mainly for the conditions related to limit transitions). For some of them, their justification comes with the proof of Lemma 2.3,

Let $\sigma$ be an $\alpha$-model and $\phi$ be a formula in $\operatorname{LTL}(\mathrm{U}, \mathrm{S})$. The Hintikka sequence for $\sigma$ and $\phi$ is an $\alpha$-sequence $H^{\sigma, \phi}$ defined as follows: for every $\beta<\alpha$,

$$
H^{\sigma, \phi}(\beta) \stackrel{\text { def }}{=}\{\psi \in \operatorname{sub}(\phi): \sigma, \beta \models \psi\} .
$$

Given a run $r: \alpha \rightarrow Q$, we write $\bmod (r): \alpha \rightarrow \mathcal{P}(\mathrm{PROP})$ to denote the $\alpha$-model $\sigma$ defined as follows: $\sigma(\beta) \stackrel{\text { def }}{=}\{p \in \mathrm{PROP}: p \in r(\beta)\}$. It is clear that if $r$ is an Hintikka sequence for $\sigma$ and $\phi$, then $\bmod (r)=\sigma$.

Now we can state the correctness lemma.

\section{Lemma 2.3.}

(I): If $\sigma, 0 \models \phi$, then the Hintikka sequence for $\sigma$ and $\phi$ is an accepting run of $\mathcal{A}_{\phi}$.

(II): If $r$ is an accepting run of $\mathcal{A}_{\phi}$, then $\bmod (r), 0 \models \phi$ and $r$ is the Hintikka sequence for $\bmod (r)$ and $\phi$.

(III): $\phi$ is satisfiable iff $\mathcal{A}_{\phi}$ has an accepting run.

Proof. First, (III) is an immediate consequence of (I) and (II).

(I): Suppose that there is a model $\sigma: \alpha \rightarrow \mathcal{P}(\mathrm{PROP})$ (with $\alpha>0$ ) such that $\sigma, 0 \models \phi$. By using $\operatorname{LTL}(\mathrm{U}, \mathrm{S})$ semantics, it is straightforward to check that $H^{\sigma, \phi}$ is accepted by $\mathcal{A}_{\phi}$.

(II): Let $r: \alpha \rightarrow Q$ be an accepting run of $\mathcal{A}_{\phi}$. Let us show by structural induction that for all $\psi \in \operatorname{sub}(\phi)$ and $\beta<\alpha$, we have $\bmod (r), \beta \models \psi$ iff $\psi \in r(\beta)$. The base case and the cases with Boolean operators in the induction step are by an easy verification. The only interesting cases in the induction step are related to the temporal operators $\mathrm{U}$ and $\mathrm{S}$. Below, let $\sigma$ be $\bmod (r)$.

Case U: $\psi=\psi_{1} \mathrm{U} \psi_{2}$.

Let us reason ad absurdum. Suppose that $\{\beta: \psi \in r(\beta)\} \neq\{\beta: \sigma, \beta \models \psi\}$. Let $\beta$ be the smallest ordinal which belongs to only one of these sets. We consider two cases: $(\sigma, \beta \models \psi$ and $\psi \notin r(\beta))$ - Case I below - or $(\psi \in r(\beta)$ and $\sigma, \beta \not \psi \psi)$ - Case II below.

Case I: Let $\gamma$ be the smallest ordinal verifying $\beta<\gamma<\alpha, \sigma, \gamma \models \psi_{2}$ and for every $\gamma^{\prime} \in(\beta, \gamma)$, we have $\sigma, \gamma^{\prime} \models \psi_{1} \wedge \neg \psi_{2}$. By induction hypothesis, $\psi_{2} \in r(\gamma)$ and for every $\gamma^{\prime} \in(\beta, \gamma),\left\{\psi_{1}, \neg \psi_{2}\right\} \subseteq r\left(\gamma^{\prime}\right)$. 
First, we are going to show that $\neg \psi \in r\left(\gamma^{\prime}\right)$ for every $\gamma^{\prime} \in[\beta, \gamma)$. This is true for $\beta$. Assume that this is true for $\beta^{\prime}$ then it is true for $\beta^{\prime}+1$ by condition (nextu). Assume that $\gamma^{\prime}$ is a limit ordinal and $\psi \notin r\left(\beta^{\prime}\right)$ for every $\beta^{\prime} \in\left[\beta, \gamma^{\prime}\right)$. Then, by condition (limu2) we obtain that $\neg \psi \in r\left(\gamma^{\prime}\right)$. Next, consider two cases:

Case a): $\gamma$ is a successor, say $\gamma=\beta^{\prime}+1$. We have $\neg \psi \in r\left(\beta^{\prime}\right)$ and $\psi_{2} \in r(\gamma)$. This contradicts condition (nextu).

Case b): $\gamma$ is a limit ordinal. In this case $\left\{\neg \psi, \psi_{1}\right\} \subseteq B_{\text {lim }}(r, \gamma)$ and $\psi_{2} \in r(\gamma)$. This contradicts condition (limu 3$)$.

Case II: Now suppose that $\psi_{1} \mathrm{U} \psi_{2} \in r(\beta)$ and $\sigma, \beta \not \forall \psi_{1} \mathrm{U} \psi_{2}$.

Case $a)$ : For every $\gamma$ such that $\gamma \in(\beta, \alpha)$, we have $\sigma, \gamma \not \models \psi_{2}\left(\psi_{2}\right.$ does not hold on $\sigma$ strictly after $\beta$ ).

By induction hypothesis, for every $\gamma \in(\beta, \alpha), \neg \psi_{2} \in r(\gamma)$. Let us show that for every $\gamma \in(\beta, \alpha),\left\{\psi_{1}, \psi_{1} \mathrm{U} \psi_{2}\right\} \subseteq r(\gamma)$.

Base case: $\gamma=\beta+1$.

By condition (nextu), $\psi_{1} \mathrm{U} \psi_{2} \in r(\beta)$ and $\neg \psi_{2} \in r(\beta+1)$ imply $\left\{\psi_{1}, \psi_{1} \mathrm{U} \psi_{2}\right\} \subseteq$ $r(\beta+1)$.

Induction step:

- if $\gamma=\gamma^{\prime}+1$, then by condition (nextu), $\psi_{1} \mathrm{U} \psi_{2} \in r\left(\gamma^{\prime}\right)$ and $\neg \psi_{2} \in r\left(\gamma^{\prime}+1\right)$ imply $\left\{\psi_{1}, \psi_{1} \mathrm{U} \psi_{2}\right\} \subseteq r\left(\gamma^{\prime}+1\right)$.

- if $\gamma$ is a limit ordinal, by induction hypothesis, $\left\{\psi_{1}, \neg \psi_{2}, \psi_{1} \mathrm{U} \psi_{2}\right\} \subseteq B_{\text {lim }}(r, \gamma)$. By condition (limu 1$),\left\{\psi_{1}, \psi_{1} \mathrm{U} \psi_{2}\right\} \subseteq r(\gamma)$ since $\psi_{2} \notin r(\gamma)$.

Consequently, if $\alpha$ is a limit ordinal, then $\left\{\psi_{1}, \neg \psi_{2}, \psi_{1} \cup \psi_{2}\right\} \subseteq B_{\text {lim }}(r, \alpha)$ which is in contradiction with the definition of $\mathcal{F}$ in $\mathcal{A}_{\phi}$. Similarly, if $\alpha=\alpha^{\prime}+1$, then $\psi_{1} \mathrm{U} \psi_{2} \in r\left(\alpha^{\prime}\right)$ which is in contradiction with the definition of $F$.

Case b): There is a minimal ordinal $\gamma \in(\beta, \alpha)$ such that $\sigma, \gamma \models \neg \psi_{1} \wedge \neg \psi_{2}$ and for every $\gamma^{\prime} \in(\beta, \gamma)$, we have $\sigma, \gamma^{\prime} \models \psi_{1} \wedge \neg \psi_{2}$. By induction hypothesis, $\left\{\neg \psi_{1}, \neg \psi_{2}\right\} \subseteq r(\gamma)$ and for every $\gamma^{\prime} \in(\beta, \gamma),\left\{\psi_{1}, \neg \psi_{2}\right\} \subseteq r\left(\gamma^{\prime}\right)$. Let us show that for every $\gamma^{\prime} \in(\beta, \gamma),\left\{\psi_{1}, \psi_{1} \cup \psi_{2}\right\} \subseteq r\left(\gamma^{\prime}\right)$.

Base case: $\gamma^{\prime}=\beta+1$.

By condition (nextu), $\psi_{1} \mathrm{U} \psi_{2} \in r(\beta)$ and $\neg \psi_{2} \in r(\beta+1)$ imply $\left\{\psi_{1}, \psi_{1} \mathrm{U} \psi_{2}\right\} \subseteq$ $r\left(\gamma^{\prime}\right)$.

Induction step:

- If $\gamma^{\prime}=\gamma^{\prime \prime}+1$, then by condition (nextu), $\psi_{1} \mathrm{U} \psi_{2} \in r\left(\gamma^{\prime \prime}\right)$ and $\neg \psi_{2} \in r\left(\gamma^{\prime \prime}+1\right)$ imply $\left\{\psi_{1}, \psi_{1} \mathrm{U} \psi_{2}\right\} \subseteq r\left(\gamma^{\prime \prime}+1\right)$.

- If $\gamma^{\prime}$ is a limit ordinal, then by induction hypothesis, $\left\{\psi_{1}, \neg \psi_{2}, \psi_{1} \mathrm{U} \psi_{2}\right\} \subseteq$ $B_{\text {lim }}\left(r, \gamma^{\prime}\right)$. By condition (limu1), $\left\{\psi_{1}, \psi_{1} \mathrm{U} \psi_{2}\right\} \subseteq r\left(\gamma^{\prime}\right)$ since $\psi_{2} \notin r\left(\gamma^{\prime}\right)$.

Consequently, if $\gamma$ is a limit ordinal, then $\left\{\psi_{1}, \neg \psi_{2}, \psi_{1} \mathrm{U} \psi_{2}\right\} \subseteq B_{\text {lim }}(r, \gamma)$ which leads to a contradiction by condition (limu 1 ). Indeed, by induction hypothesis, $\left\{\neg \psi_{1}, \neg \psi_{2}\right\} \subseteq r(\gamma)$. Similarly, if $\gamma=\gamma^{\prime}+1$, then $\psi_{1} \mathrm{U} \psi_{2} \notin r\left(\gamma^{\prime}\right)$ which leads to a contradiction by condition (nextu).

Case S: $\psi=\psi_{1} \mathrm{~S} \psi_{2}$.

Let us reason ad absurdum. Suppose that $\{\beta: \psi \in r(\beta)\} \neq\{\beta: \sigma, \beta \models \psi\}$. Let $\beta$ be the smallest ordinal that belongs to only one of these sets. Again, we distinguish 
two cases, namely either $(\sigma, \beta \models \psi$ and $\psi \notin r(\beta))$ - Case I below - or $(\psi \in r(\beta)$ and $\sigma, \beta \not \psi \psi)$ - Case II below.

Case I: So $\beta>0$ and there is $\gamma \in[0, \beta)$ such that $\sigma, \gamma \models \psi_{2}$ and for every $\gamma^{\prime} \in(\gamma, \beta)$, we have $\sigma, \gamma^{\prime} \models \psi_{1}$. By induction hypothesis, $\psi_{2} \in r(\gamma)$ and for every $\gamma^{\prime} \in(\gamma, \beta)$, $\psi_{1} \in r\left(\gamma^{\prime}\right)$. Observe that for every $\gamma^{\prime} \in(\gamma, \beta)$, we have $\sigma, \gamma^{\prime} \models \psi$ and $\psi \in r\left(\gamma^{\prime}\right)$ ( $\beta$ is minimal).

- If $\beta=\beta^{\prime}+1$ then by condition (nexts) $\psi_{2} \notin r\left(\beta^{\prime}\right)$ and $\left\{\psi_{1}, \psi_{1} \mathrm{~S} \psi_{2}\right\} \nsubseteq r\left(\beta^{\prime}\right)$. If $\gamma=\beta^{\prime}$, then this leads to a contradiction since $\psi_{2} \in r(\gamma)$. Similarly, if $\gamma<\beta^{\prime}$, then $\psi \notin r\left(\beta^{\prime}\right)$ since $\psi_{1} \in r\left(\beta^{\prime}\right)$. Since $\sigma, \beta^{\prime} \models \psi_{1} \mathrm{~S} \psi_{2}$, this leads to a contradiction by the minimality of $\beta$.

- If $\beta$ is a limit ordinal, then by condition (lims) either $\psi_{1} \notin B_{l i m}(r, \beta)$ or $\psi_{1} \mathrm{~S} \psi_{2} \notin$ $B_{\text {lim }}(r, \beta)$. By induction hypothesis, $\psi_{1} \in B_{\text {lim }}(r, \beta)$. Hence, there is $\beta^{\prime} \in(\gamma, \beta)$ such that $\psi_{1} \mathrm{~S} \psi_{2} \notin r\left(\beta^{\prime}\right)$, which is in contradiction with the minimality of $\beta$.

\section{Case II:}

Case $a)$ : For every $\gamma \in[0, \beta), \sigma, \beta \not \models \psi_{2}$.

By induction hypothesis, for every $\gamma \in[0, \beta), \psi_{2} \notin r(\gamma)$. Moreover, for every $\gamma \in[0, \beta)$, we have $\sigma, \beta \not \forall \psi_{1} \mathrm{~S} \psi_{2}$.

- If $\beta=\beta^{\prime}+1$ then by condition (nexts), $\left\{\psi_{1}, \psi_{1} \mathrm{~S} \psi_{2}\right\} \subseteq r\left(\beta^{\prime}\right)$ which leads to a contradiction by minimality of $\beta$.

- If $\beta$ is a limit ordinal, then $\left\{\psi_{1}, \psi_{1} \mathrm{~S} \psi_{2}\right\} \subseteq B_{\text {lim }}(r, \beta)$ by condition ( $\left.\lim _{\mathrm{S}}\right)$. Hence, for some $\beta^{\prime}<\beta, \psi_{1} \mathrm{~S} \psi_{2} \in r\left(\beta^{\prime}\right)$, which leads again to a contradiction by the minimality of $\beta$.

- If $\beta=0$, then we also have a contradiction since $r(0)$ does not contain any since formulae. Observe that in the previous case analyses with ordinals, the case " 0 " has been irrelevant.

Case b): $\sigma, \beta \not \forall \psi$ and not a).

Remember that $\psi \in r(\beta)$. There is $\beta^{\prime}<\beta$ such that $\sigma, \beta^{\prime} \not \neq \psi_{1}$. Otherwise, by induction hypothesis and by not a), we have $\sigma, \beta \models \psi$, a contradiction.

Case b.1: There is a maximal position $\gamma<\beta$ such that $\sigma, \gamma \not \models \psi_{1}$.

For every $\gamma^{\prime} \in(\gamma, \beta)$, we have $\sigma, \gamma \not \models \psi_{2}$, otherwise $\sigma, \beta \models \psi$ which would lead to a contradiction. Let us show by transfinite induction that for every $\gamma^{\prime} \in(\gamma, \beta]$, $\psi \notin r\left(\gamma^{\prime}\right)$.

Base case: $\gamma^{\prime}=\gamma+1$.

$\neg \psi_{1}, \neg \psi_{2} \in r(\gamma)$ imply by condition (nexts) that $\psi \notin r\left(\gamma^{\prime}\right)$.

Induction step:

- If $\gamma^{\prime}=\gamma^{\prime \prime}+1$, then $\neg \psi_{2}, \neg \psi \in r\left(\gamma^{\prime \prime}\right)$ by induction hypothesis. By condition (nexts) $\psi \notin r\left(\gamma^{\prime}\right)$.

- If $\gamma^{\prime}$ is a limit ordinal, then $\neg \psi \in B_{\text {lim }}\left(r, \gamma^{\prime}\right)$ and by condition (lims), $\psi \notin r\left(\gamma^{\prime}\right)$. Hence, $\psi \notin r(\beta)$, which leads to a contradiction.

Case b.2 There is no maximal position $\gamma<\beta$ such that $\sigma, \gamma \not \models \psi_{1}$ (the most delicate case).

Consequently, there is a unique position $\gamma \leq \beta$ such that for every $\gamma^{\prime}<\gamma$, there is $\gamma^{\prime}<\gamma^{\prime \prime}<\gamma$ verifying $\neg \psi_{1} \in r\left(\gamma^{\prime \prime}\right)$. This means that

- for every $\gamma^{\prime} \in[\gamma, \beta], \psi_{1} \in r\left(\gamma^{\prime}\right)$,

- $\psi_{1} \notin B_{l i m}(r, \gamma)$ and, 
- by condition (lims) $\psi \notin r(\gamma)$.

Moreover, for every $\gamma^{\prime} \in(\gamma, \beta), \neg \psi_{2} \in r\left(\gamma^{\prime}\right)$ otherwise by induction hypothesis, $\sigma, \beta \models \psi$, which would lead to a contradiction. Let us show by transfinite induction that for every $\gamma^{\prime} \in(\gamma, \beta], \psi \notin r\left(\gamma^{\prime}\right)$.

Base case: $\gamma^{\prime}=\gamma+1$.

$\neg \psi, \neg \psi_{2} \in r(\gamma)$ imply by condition (nexts) $\psi \notin r\left(\gamma^{\prime}\right)$.

Induction step:

- If $\gamma^{\prime}=\gamma^{\prime \prime}+1$, then $\neg \psi_{2}, \neg \psi \in r\left(\gamma^{\prime \prime}\right)$ by induction hypothesis. By condition (nexts) $\psi \notin r\left(\gamma^{\prime}\right)$.

- If $\gamma^{\prime}$ is a limit ordinal, then $\neg \psi \in B_{\text {lim }}\left(r, \gamma^{\prime}\right)$ and by condition (lims), $\psi \notin r\left(\gamma^{\prime}\right)$. Hence, $\psi \notin r(\beta)$, which leads to a contradiction.

\section{Short Run Properties}

In this section, we establish pumping arguments that are useful to show that

- in order to check the satisfiability status of the formula $\phi$, there is no need to consider models of length greater than $\omega^{|\phi|+2}$,

- simple ordinal automata cannot distinguish ordinals with identical tails (defined below precisely with the notion of truncation).

Let $\mathcal{A}$ be a simple ordinal automaton and $Y$ be a subset of its basis. $Y$ is said to be present in $\mathcal{A}$ iff either there is a limit transition of the form $\langle Y, q\rangle$ in $\mathcal{A}$ or $Y \in \mathcal{F}$. Given a set $Y$ present in $\mathcal{A}$, its weight, noted weight $(Y)$, is the maximal $l$ such that $Y_{1} \subset Y_{2} \subset \cdots \subset Y_{l}$ is a sequence of present subsets in $\mathcal{A}$ and $Y_{1}=Y$ ( $\subset$ denotes proper subset inclusion). Obviously, weight $(Y) \leq \operatorname{card}(B)+1$.

Given a path $r: \alpha \rightarrow Q$ in $\mathcal{A}$ with a limit ordinal $\alpha \geq \omega$, its weight, noted weight $(r)$, is the maximal value in the set

$$
\left\{\operatorname{weight}\left(B_{\text {lim }}(r, \beta)\right): \beta<\alpha, \beta \text { is a limit ordinal }\right\} \cup\left\{\operatorname{weight}\left(B_{\text {lim }}(r)\right)\right\}
$$

When $\alpha$ is a successor ordinal, the maximal value is computed only from the first set of the above union. By convention, if a path has no limit transition, then its weight is zero (equivalently, its length is strictly less than $\omega+1$ ). Furthermore, we write all $(r)$ to denote the set

$$
\operatorname{all}(r) \stackrel{\text { def }}{=} \bigcap_{\beta<\alpha} r(\beta)
$$

that corresponds to the set of elements from the basis that are present in all locations of the run $r$. Let $r, r^{\prime}$ be two paths of respective length $\alpha$ and $\alpha^{\prime}$. We say that $r$ and $r^{\prime}$ are congruent, written $r \sim r^{\prime}$, iff the conditions below are meet:

(1) $r(0)=r^{\prime}(0)$.

(2) Either both $\alpha$ and $\alpha^{\prime}$ are successor ordinals and $r(\alpha-1)=r^{\prime}\left(\alpha^{\prime}-1\right)$ or both $\alpha$ and $\alpha^{\prime}$ are limit ordinals and $B_{\text {lim }}(r)=B_{\text {lim }}\left(r^{\prime}\right)$.

(3) $\operatorname{all}(r)=\operatorname{all}\left(r^{\prime}\right)$.

Let $r_{1}$ be a path of length $\alpha$ and $r_{2}$ be a path of length $\beta$ such that if $\alpha$ is a limit ordinal then $\left\langle B_{\text {lim }}\left(r_{1}\right), r_{2}(0)\right\rangle \in \delta_{\text {lim }}$ otherwise $r_{1}(\alpha-1)=r_{2}(0)$. The concatenation $r_{1} \cdot r_{2}$ is the path $r$ of length $\alpha+\beta$ such that for $\gamma \in[0, \alpha), r(\gamma)=r_{1}(\gamma)$ and for $\gamma \in[0, \beta)$, $r(\alpha+\gamma)=r_{2}(\gamma)$. For every ordinal $\alpha$, the concatenation of $\alpha$-sequences of paths is defined 
similarly. The relation $\sim$ is a congruence for the concatenation operation on paths as stated in details below.

\section{Lemma 3.1.}

(I): Let $r \cdot r_{0} \cdot r^{\prime}$ and $r_{1}$ be two paths such that $r_{0} \sim r_{1}$. Then, $r \cdot r_{1} \cdot r^{\prime}$ is a path that is congruent to $r \cdot r_{0} \cdot r^{\prime}$.

(II): Let $r_{0}^{0}, r_{0}^{1}, r_{0}^{2}, \ldots$ and $r_{1}^{0}, r_{1}^{1}, r_{1}^{2}, \ldots$ be two $\omega$-sequences of pairwise consecutive paths such that for $i \geq 0, r_{0}^{i} \sim r_{1}^{i}$ and their length is a successor ordinal. If $r \cdot\left(r_{0}^{0} \cdot r_{0}^{1} \cdot r_{0}^{2}\right.$. $\ldots) \cdot r^{\prime}$ is a path, then it is congruent to $r \cdot\left(r_{1}^{0} \cdot r_{1}^{1} \cdot r_{1}^{2} \cdot \ldots\right) \cdot r^{\prime}$.

The proof of the above lemma is by an easy verification but observe that for the proof of (II) the third set of equalities from the definition of the congruence $\sim$ ensures that $r \cdot\left(r_{0}^{0} \cdot r_{0}^{1} \cdot r_{0}^{2} \cdot \ldots\right) \cdot r^{\prime}$ is a path.

Lemma 3.2. Let $r: \alpha \rightarrow Q$ be a path in $\mathcal{A}$ for some countable ordinal $\alpha$ such that if $\alpha$ is a limit ordinal, then $B_{\text {lim }}(r)$ is present in $\mathcal{A}$. Then, there is a path $r^{\prime}: \alpha^{\prime} \rightarrow Q$ for $\alpha^{\prime}<\omega^{\max }(1, \operatorname{weight}(r))+1$ such that $r \sim r^{\prime}$ and weight $\left(r^{\prime}\right) \leq \operatorname{weight}(r)$.

Proof. The proof is by induction on the weight of the paths. When the weight of the path is zero, $r^{\prime}=r$ already satisfies the condition $r \sim r^{\prime}$. We only treat below the cases with paths of length some limit ordinals. The case with paths of length some successor ordinals is similar. All the runs $r^{\prime}$ built below satisfy that weight $\left(r^{\prime}\right) \leq$ weight $(r)$ for the following reasons. Indeed, no additional limit transitions are applied when building $r^{\prime}$ from $r$ and when $r$ is of length some limit ordinal, $B_{l i m}(r)=B_{l i m}\left(r^{\prime}\right)$. Hence, below we shall not further emphasize weight $\left(r^{\prime}\right) \leq$ weight $(r)$.

Base case 1: weight $(r)=1$ and $\alpha=\omega^{2}$.

There is $n \geq 0$ such that

(1) for every $a \in B \backslash$ all $(r)$, there is $\gamma \leq \omega \cdot n$ such that $a \notin r(\gamma)$,

(2) $\operatorname{all}\left(r_{\geq \omega \cdot n}\right)=B_{\text {lim }}(r)$.

The first condition states that if $a$ does not belong to all $(r)$, then this is already witnessed by $r_{\leq \omega \cdot n}$. Furthermore, in general all $\left(r_{\geq \omega \cdot k}\right) \subseteq B_{\text {lim }}(r)$ but the second condition above states that for $n$ large enough, we can obtain $B_{l i m}(r) \subseteq \operatorname{all}\left(r_{\geq \omega \cdot n}\right)$.

Hence, $\operatorname{all}\left(r_{\leq \omega \cdot n}\right)=\operatorname{all}(r)$ and $B_{l i m}\left(r_{\geq \omega \cdot(n+1)}\right)=B_{l i m}(r)$. Besides, $B_{l i m}(r)$ is present in $\mathcal{A}$. Let $Y_{i}=B_{\text {lim }}\left(r_{\leq \omega \cdot i}\right)$ for $i \geq n+1$. By construction of $n$, for all $i \geq n+1, B_{\text {lim }}(r) \subseteq Y_{i}$. Moreover, weight $\left(B_{l i m}(r)\right)=1$. Hence, for all $i \geq n+1, Y_{i}=B_{l i m}(r)$. Consequently, $r^{\prime}: \omega \cdot(n+1) \rightarrow Q$ with $r^{\prime}(\beta)=r(\beta)$ for $\beta<\omega \cdot(n+1)$ verifies $r \sim r^{\prime}$. In order to show that $\operatorname{all}(r)=\operatorname{all}\left(r^{\prime}\right)$ it is sufficient to observe that $\operatorname{all}(r) \subseteq \operatorname{all}\left(r^{\prime}\right)$ since $r^{\prime}$ contains less locations than $r$ and $\operatorname{all}\left(r_{\leq \omega \cdot n}^{\prime}\right)=\operatorname{all}(r)$.

Base case 2: weight $(r)=1$ and $\alpha=\omega^{2} \times \beta$.

The proof is by transfinite induction. The base case with $\beta=1$ is actually the above base case 1. Now suppose that $\alpha=\omega^{2} \times(\beta+1)$. By induction hypothesis and by the base case 1, there are paths $r^{\prime}: \gamma \rightarrow Q$ and $r^{\prime \prime}: \gamma^{\prime} \rightarrow Q$ such that $r^{\prime} \sim r_{<\omega^{2} \times \beta}, r^{\prime \prime} \sim r_{\geq \omega^{2} \times \beta}$ and $\gamma+\gamma^{\prime}<\omega^{2}$. Consequently, the concatenation of $r^{\prime}$ and $r^{\prime \prime}$ provides a path satisfying the adequate conditions.

Now suppose that $\alpha=\omega^{2} \times \beta$ where $\beta$ is a limit ordinal. Since $\alpha$ is countable, there is an increasing sequence $\left(\beta_{i}\right)_{i \in \mathbb{N}}$ of ordinals strictly smaller than $\beta$ such that $\beta_{0}=0$ and $\beta=$ $\lim \beta_{i}$ (see e.g. [Ros82, Theorem 3.36]). Observe that for every $i, \beta_{i+1}-\beta_{i}<\beta$. Hence, for every $i$, by induction hypothesis, there is a path $r_{i}^{\prime}: \gamma_{i} \rightarrow Q$ such that $r_{i}^{\prime} \sim r_{\left[\omega^{2} \times \beta_{i}, \omega^{2} \times \beta_{i+1}\right)}$ 
and $\gamma_{i}<\omega^{2}$. Consequently, $r_{0}^{\prime} \cdot r_{1}^{\prime} \cdot r_{2}^{\prime} \cdots$ is a path of length at most $\omega^{2}$ congruent to $r$ by Lemma 3.1 (the length may be exactly $\omega^{2}$ ). By using again arguments from the base case 1 , we obtain a path that satisfies the adequate conditions.

Base case 3: weight $(r)=1$ and $\alpha=\omega^{2} \times \beta+\omega \times n(n \in \mathbb{N})$.

The existence of a path satisfying the adequate conditions is an immediate consequence of the base case 2 .

Induction case.

Case 1: $\alpha=\omega^{\text {weight }(r)+1}$.

There is $n \geq 0$ such that

(1) for every $a \in B \backslash \operatorname{all}(r)$, there is $\gamma \leq \omega^{\operatorname{weight}(r)} \cdot n$ such that $a \notin r(\gamma)$,

(2) $\operatorname{all}\left(r_{\geq \omega^{\text {weight }(r) \cdot n}}\right)=B_{\text {lim }}(r)$.

Hence, $\operatorname{all}\left(r_{\leq \omega^{\text {weight }(r) \cdot n}}\right)=\operatorname{all}(r)$ and $B_{l i m}\left(r_{\geq \omega^{\text {weight }(r) \cdot(n+1)}}\right)=B_{l i m}(r)$. Besides, $B_{l i m}(r)$ is present in $\mathcal{A}$ and weight $\left(B_{\text {lim }}(r)\right) \leq$ weight $(r)$. If there is a limit ordinal $\beta \in\left[\omega^{\operatorname{weight}(r)} \cdot n, \alpha\right)$ such that $B_{\text {lim }}\left(r_{<\beta}\right)=B_{\text {lim }}(r)$, then $r^{\prime}: \beta \rightarrow Q$ with $r^{\prime}(\gamma)=r(\gamma)$ for $\gamma<\beta$ verifies the required conditions. Otherwise, suppose that for every limit ordinal $\beta \in\left[\omega^{\operatorname{weight}(r)} \cdot n, \alpha\right)$, we have $B_{\text {lim }}\left(r_{<\beta}\right) \neq B_{\text {lim }}(r)$. By construction of $n$, for every limit ordinal $\beta$ in $\left[\omega^{\operatorname{weight}(r)} \cdot n, \alpha\right)$, $B_{\text {lim }}(r) \subset B_{\text {lim }}\left(r_{<\beta}\right)$. By induction hypothesis, for every $i>n$, there is a path $r_{i}^{\prime}: \gamma_{i} \rightarrow Q$

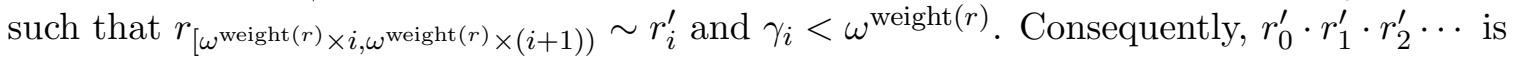
a path of length less than $\omega^{\text {weight }(r)}$ that is congruent to $r$ by Lemma 3.1 .

Case 2: $\alpha=\omega^{\text {weight }(r)+1} \times \beta$.

The proof is by transfinite induction as in the base case 2. Indeed, suppose that $\alpha=$ $\omega^{\text {weight }(r)+1} \times(\beta+1)$. There are paths $r^{\prime}: \gamma \rightarrow Q$ and $r^{\prime \prime}: \gamma^{\prime} \rightarrow Q$ such that $r^{\prime} \sim$

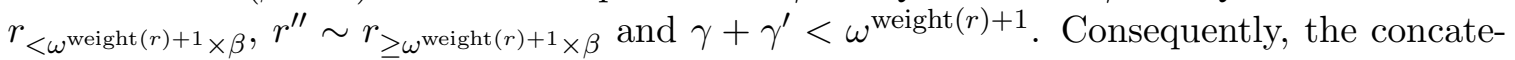
nation of $r^{\prime}$ and $r^{\prime \prime}$ provides a path satisfying the adequate conditions.

Now suppose that $\alpha=\omega^{\text {weight }(r)+1} \times \beta$ where $\beta$ is a limit ordinal. Hence, there is an increasing sequence $\left(\beta_{i}\right)_{i \in \mathbb{N}}$ of ordinals strictly smaller than $\beta$ such that $\beta_{0}=0$ and $\beta=\lim \beta_{i}$ (see e.g. Ros82, Theorem 3.36]). Observe that for every $i, \beta_{i+1}-\beta_{i}<\beta$. Hence, for every $i$, by induction hypothesis, there is a path $r_{i}^{\prime}: \gamma_{i} \rightarrow Q$ such that $r_{i}^{\prime} \sim$ $r_{\left[\omega^{\text {weight }(r)+1} \times \beta_{i}, \omega^{\text {weight }(r)+1} \times \beta_{i+1}\right)}$ and $\gamma_{i}+1<\omega^{\text {weight }(r)+1}$. Consequently, $r_{0}^{\prime} \cdot r_{1}^{\prime} \cdot r_{2}^{\prime} \cdots$ is a path of length less than $\omega^{\text {weight }(r)+1}$ congruent to $r$ by Lemma 3.1 (the lenght may be equal to $\left.\omega^{\text {weight }(r)+1}\right)$. By using the case 1 in the induction step, we can get a path that satisfies the adequate conditions.

Case 3: $\alpha=\omega^{\text {weight }(r)+1} \times \beta+\omega^{\operatorname{weight}(r)} \times n_{\text {weight }(r)}+\cdots+\omega^{1} \times n_{1}$ with $n_{\text {weight }(r)}, \ldots$, $n_{1} \in \mathbb{N}$.

The existence of a path satisfying the required conditions is an immediate consequence of the case 2 .

Lemma 3.2 below states a crucial property for most of complexity results established in the sequel. Indeed, for usual ordinal automata, it is not possible to get this polynomial bound as an exponent of $\omega$ for the length of the short paths. Actually, the exponent is linear in the cardinal of its basis and can be logarithmic in the number of locations for large automata. By combination of Lemma 2.3 and Lemma 3.2, we obtain the following interesting result.

Corollary 3.3. If $\phi$ is satisfiable, then $\phi$ has an $\alpha$-model with $\alpha<\omega^{|\phi|+2}$. 
For $n \in \mathbb{N}$, let trunc $n$ be the function that assigns to every ordinal $\alpha>0$ an ordinal in $\left(0, \omega^{n} 2\right)$ as follows. $\alpha$ can be written in the form $\alpha=\omega^{n} \gamma+\beta$ with $\beta \in\left[0, \omega^{n}\right)$. Then $\operatorname{trunc}_{n}(\alpha)=\omega^{n} \times \min (\gamma, 1)+\beta$. For instance $\operatorname{trunc}_{2}\left(\omega^{3}\right)=\omega^{2}, \operatorname{trunc}_{2}\left(\omega^{2}+\omega\right)=\omega^{2}+\omega$ and $\operatorname{trunc}_{2}\left(\omega^{2} \times 2\right)=\omega^{2}$. The ordinals $\alpha, \beta$ are $n$-equivalent, written $\alpha \approx_{n} \beta$, def $\operatorname{trunc}_{n}(\alpha)=\operatorname{trunc}_{n}(\beta)$.

Lemma 3.4. Let $\mathcal{A}$ be a simple ordinal automaton.

(I): If $r$ is a path of length $\omega^{\text {weight }(r)+1} \times \alpha$ for some countable ordinal $\alpha>0$, then there is a path $r^{\prime}$ of length $\omega^{\text {weight }(r)+1}$ such that $r \sim r^{\prime}$ and weight $\left(r^{\prime}\right) \leq \operatorname{weight}(r)$.

(II): If a path $r$ has length $\omega^{\text {weight }(r)+1}$ and weight $(r) \geq 1$, then for every ordinal $\alpha>0$, there is a path $r^{\prime}$ of length $\omega^{\text {weight }(r)+1} \times \alpha$ such that $r \sim r^{\prime}$ and weight $\left(r^{\prime}\right) \leq \operatorname{weight}(r)$.

(III): If $r$ is a path of length some countable ordinal $\alpha$ and $\beta \approx_{\operatorname{card}(B)+2} \alpha$, then there is a path $r^{\prime}$ of length $\beta$ such that $r \sim r^{\prime}$.

Only in (I), the ordinal $\alpha$ is supposed to be countable.

Proof. (III) is a direct consequence of (I) and (II). Indeed, suppose $\alpha=\omega^{\operatorname{card}(B)+2} \gamma_{0}+\gamma_{1}$ and $\beta=\omega^{\operatorname{card}(B)+2} \gamma_{0}^{\prime}+\gamma_{1}^{\prime}$ with $\gamma_{1}=\gamma_{1}^{\prime} \in\left[0, \omega^{\operatorname{card}(B)+2}\right)$, and $\gamma_{0} \geq 1$ iff $\gamma_{0}^{\prime} \geq 1$. If $\gamma_{0}=\gamma_{0}^{\prime}=0$, then $\alpha=\beta$ and we are done. Otherwise (weight $(r) \geq 1$ ), let $K>0$ such that $K+\operatorname{weight}(r)=\operatorname{card}(B)+2$. Since weight $(r) \leq \operatorname{card}(B)+1$ such a value $K$ exists and therefore (I) can be applied. There is a run $r^{\prime}$ such that $r^{\prime} \sim r_{\leq \omega^{\operatorname{card}(B)+2} \gamma_{0}}$ and $r^{\prime}$ is of length $\omega^{\text {weight }(r)+1}$ by (I). If weight $\left(r^{\prime}\right) \neq$ weight $(r)$, then we apply again (I) on $r^{\prime}$ in order to obtain a run $r^{\prime \prime}$ such that $r^{\prime \prime} \sim r^{\prime}, r^{\prime \prime}$ is of length $\omega^{\text {weight }\left(r^{\prime}\right)+1}$. If again $\operatorname{weight}\left(r^{\prime \prime}\right) \neq \operatorname{weight}\left(r^{\prime}\right)$, we cannot repeat this process more than $\operatorname{card}(B)+1$ times. Eventually, we obtain a run $r_{0}$ such that $r_{0} \sim r_{\leq \omega^{\operatorname{card}(B)+2} \gamma_{0}}$ and $r_{0}$ is of length $\omega^{\text {weight }\left(r_{0}\right)+1}$. By (II), there is a run $r_{1}$ such that $r_{1} \sim r_{0}$ and $r_{1}$ is of length $\omega^{\operatorname{card}(B)+2} \gamma_{0}^{\prime}$ by (II). Consequently, $r_{1} \cdot r_{\geq \omega^{\operatorname{card}(B)+2} \gamma_{0}} \sim r$ and $r_{1} \cdot r_{\geq \omega^{\operatorname{card}(B)+2} \gamma_{0}}$ is of length $\beta$.

(I): The proof is by transfinite induction on $\alpha$. Again, all the runs $r^{\prime}$ built below satisfy that weight $\left(r^{\prime}\right) \leq$ weight $(r)$ for the following reasons. Indeed, no additional limit transitions are applied when building $r^{\prime}$ from $r$ and when $r$ is of length some limit ordinal, $B_{\text {lim }}(r)=B_{\text {lim }}\left(r^{\prime}\right)$. Hence, below we shall not further emphasize weight $\left(r^{\prime}\right) \leq$ weight $(r)$. We behave similarly for the proof of (II).

Observe that the run $r$ cannot be of length $\omega$. In the sequel, we assume that weight $(r) \geq 1$. The base case with $\alpha=1$ is immediate. Suppose that the induction assertion holds true for $\alpha$ and let us show that it holds true for $\alpha+1$. By Lemma 3.2, there is a run $r^{\prime}$ of length strictly less than $\omega^{\text {weight }(r)+1}$ such that $r^{\prime} \sim r_{<\omega^{\text {weight }(r)+1} \times \alpha}$.

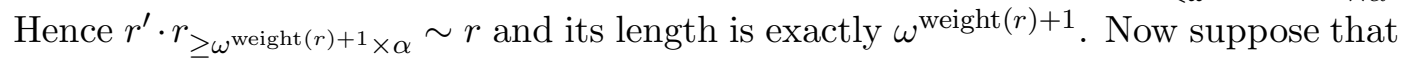
$\alpha$ is a limit ordinal and for every smaller ordinal, the property holds true. Let $r$ be a run of length $\omega^{\text {weight }(r)+1} \times \alpha$. There exists an increasing sequence $\left(\alpha_{i}\right)_{i \in \mathbb{N}}$ with $\alpha_{0}=0$ and $\alpha=\lim \alpha_{i}$ (see e.g. [Ros82, Theorem 3.36]). For $i \geq 0$, let $\alpha_{i}^{\prime}$ be $\omega^{\text {weight }(r)+1} \alpha_{i}+\omega^{\text {weight }(r)}$. Observe that $\alpha_{i}^{\prime}-\omega^{\text {weight }(r)+1} \alpha_{i}=\omega^{\text {weight }(r)}$ and $\omega^{\text {weight }(r)+1} \alpha_{i}<\alpha_{i}^{\prime}<\omega^{\text {weight }(r)+1} \alpha_{i+1}$. For $i \geq 0$, let $\beta_{i}$ be $\omega^{\text {weight }(r)+1} \times \alpha_{i}$. For every $i \geq 0$, let $r_{i}$ be the path $r_{\left[\alpha_{i}^{\prime}, \beta_{i+1}\right)}$. By Lemma 3.2. for every $j \geq 0$, there is a path $r_{j}^{\prime}$ congruent to $r_{j}$ of length strictly less than $\omega^{\operatorname{weight}(r)+1}$ and weight $\left(r_{j}^{\prime}\right) \leq \operatorname{weight}\left(r_{j}\right)$. 
Let $r^{\prime}$ be the run $r_{\left[\beta_{0}, \alpha_{0}^{\prime}\right)} r_{0}^{\prime} r_{\left[\beta_{1}, \alpha_{1}^{\prime}\right)} r_{1}^{\prime} r_{\left[\beta_{2}, \alpha_{2}\right)} r_{2}^{\prime} \ldots$. The path $r^{\prime}$ is exactly of length $\omega^{\text {weight }(r)+1}$ and it is congruent to $r$.

The proof is by double induction on the weight and on $\alpha$.

Base case: weight $(r)=1$.

Let $f:[\mathbb{N}]^{2} \rightarrow Q \times \mathcal{P}(Q) \times Q$ be the function whose domain is made of unordered pairs $\{i, j\}$ of natural numbers (say, $i<j$ ) such that

$$
f(\{i, j\}) \stackrel{\text { def }}{=}\left\langle r(\omega \times i), B_{l i m}\left(r_{[\omega \times i, \omega \times j)}\right), r(\omega \times j)\right\rangle
$$

By Ramsey's Theorem (see e.g. [Ram30, Ros82]), there is an infinite set $Y \subseteq \mathbb{N}$ such that $f$ restricted to $[Y]^{2}$ is constant. Hence, there is a value $\left\langle q^{\star}, A, q^{\star}\right\rangle$ and an infinite sequence $0 \leq i_{0}<i_{1}<i_{2}<\cdots$ such that for every $k \geq 0$, we have $f\left(\left\{i_{k}, i_{k+1}\right\}\right)=\left\langle q^{\star}, A, q^{\star}\right\rangle$. Observe that $A=B_{\text {lim }}(r)$ and for every $k$, we also have $B_{l i m}(r) \subseteq B_{l i m}\left(r_{\left[\omega \times i_{k}, \omega \times i_{k+1}\right)}\right)$. Since weight $(r)=1$, we get that $B_{\text {lim }}(r)=$ $B_{\text {lim }}\left(r_{\left[\omega \times i_{k}, \omega \times i_{k+1}\right)}\right)$.

Let us come back to the proof by induction. The base case with $\alpha=1$ is immediate. Suppose that the property holds true for $\alpha$ and let us show that it holds true for $\alpha+1$. By induction hypothesis, there is a path $r^{\prime}$ congruent to $r$ of length $\omega^{2} \times \alpha$. Since $r_{\geq \omega \times i_{0}}$ is also a path of length $\omega^{2}, r^{\prime} \cdot r_{\geq \omega \times i_{0}}$ is a path $\left(B_{l i m}\left(r_{<\omega \times i_{0}}\right)=A\right)$, it is congruent to $r$ and its length is precisely $\left(\omega^{2} \times \alpha\right)+\omega^{2}$.

Now suppose that $\alpha$ is a limit ordinal and for every smaller ordinal, the property holds true. There exists a strictly increasing sequence $\left(\alpha_{i}\right)_{i \in \mathbb{N}}$ with $\alpha_{0}=0$ and $\alpha=\lim \alpha_{i}$. By the induction hypothesis there is a run $r_{j}$ of length $\omega^{2} \times\left(\alpha_{j+1}-\alpha_{j}\right)$ congruent to $r_{\geq \omega \times i_{j}}\left(r_{\geq \omega \times i_{j}}\right.$ is also of length $\left.\omega^{2}\right)$. Then, $r_{0} \cdot r_{1} \cdot r_{2} \cdots$ is congruent to $r$ and it is of length $\omega^{2} \times \alpha$. Observe that $B_{l i m}\left(r_{0} \cdot r_{1} \cdot r_{2} \cdots\right)$ is precisely $A$ that is equal to $B_{\text {lim }}(r)$, as stated above.

Induction step:: weight $(r)>1$ and the property holds for all the paths of weight strictly less than weight $(r)$.

The base case with $\alpha=1$ is immediate.

- Suppose that the property holds true for $\alpha$ and let us show that it holds true for $\alpha+1$. As in the base case, we define a coloring function $f$ such that we color the interval with endpoints at positions of the form $\omega^{\text {weight }(r)} \times n$. Similarly to the base case, there is a triple $\left\langle q^{\star}, A, q^{\star}\right\rangle$ and a sequence $0 \leq i_{0}<i_{1}<i_{2}<\cdots$ such that for every $k \geq 0, f\left(\left\{i_{k}, i_{k+1}\right\}\right)=\left\langle q^{\star}, A, q^{\star}\right\rangle$. If there is $\beta<\omega^{\operatorname{weight}(r)+1}$ such that $B_{\text {lim }}\left(r_{<\beta}\right)=B_{\text {lim }}(r)$ then by induction hypothesis, there is $r^{\prime} \sim r$ such that $r^{\prime}$ is of length $\omega^{\text {weight }(r)+1} \times \alpha$ and $B_{\text {lim }}\left(r^{\prime}\right)=B_{l i m}\left(r_{<\beta}\right)$. Hence $r^{\prime} \cdot r_{\geq \beta}$ is a path, $r^{\prime} \cdot r \geq \beta \sim r$ and its length is $\omega^{\text {weight }(r)+1} \times(\alpha+1)$. If there is no such an ordinal $\beta$, for every limit ordinal $\beta \in\left[\omega^{\text {weight }(r)} \times\left(i_{1}-1\right), \omega^{\text {weight }(r)} \times i_{1}\right), B_{\text {lim }}(r) \subset B_{\text {lim }}\left(r_{\beta}\right)$ since $A \subset B_{\text {lim }}\left(r_{<\beta}\right)$. Hence $W=\operatorname{weight}\left(r_{\left[\omega^{\operatorname{weight}}(r) \times\left(i_{1}-1\right), \omega^{\operatorname{weight}(r)} \times i_{1}\right)}\right)<\operatorname{weight}(r)$. By the induction hypothesis there is a run $r^{\prime} \sim r_{\left[\omega^{\text {weight }}(r) \times\left(i_{1}-1\right), \omega^{\text {weight }(r)} \times i_{1}\right)}$ of length $\omega^{W+1} \times\left(\omega^{(\operatorname{weight}(r)-W)} \times \alpha\right)$, that is of length $\omega^{\text {weight }(r)+1} \times \alpha$ by associativity of multiplication. Hence $r_{<\operatorname{weight}(r) \times\left(i_{1}-1\right)} \cdot r^{\prime} \cdot r_{\geq \omega^{\operatorname{weight}}(r) \times i_{1}} \sim r$ and it is of length $\omega^{\text {weight }(r)+1} \times(\alpha+1)$.

- Now suppose that $\alpha$ is a limit ordinal and for every smaller ordinal, the property holds true. There exists a strictly increasing sequence $\left(\alpha_{i}\right)_{i \in \mathbb{N}}$ with $\alpha_{0}=0$ and $\alpha=\lim \alpha_{i}$. As above, a triple of the form $\left\langle q^{\star}, A, q^{\star}\right\rangle$ and an $\omega$-sequence $i_{0}<$ 
$i_{1}<i_{2}<\ldots$ can be defined. Observe that for every $k \geq 1$, for every limit ordinal $\beta \in\left[\omega^{\text {weight }(r)} \times\left(i_{k}-1\right), \omega^{\text {weight }(r)} \times i_{k}\right), B_{l i m}(r) \subseteq B_{l i m}(r, \beta)$ since $A=B_{\text {lim }}(r)$ and $A \subseteq B_{\text {lim }}(r, \beta)$. Hence the weight of $r_{\left[\omega^{\text {weight }}(r) \times\left(i_{k}-1\right), \omega^{\text {weight }(r)} \times i_{k}\right)}$, noted $W_{k}$, is less or equal to weight $(r)$. By induction hypothesis, for every $k \geq 1$, there is a path $r_{k} \sim$ $r_{\left[\omega^{\text {weight }(r)} \times\left(i_{k}-1\right), \omega^{\text {weight }(r)} \times i_{k}\right)}$ of length $\omega^{W_{k}+1} \times\left(\omega^{\left(\text {weight }(r)-W_{k}\right)} \times\left(\alpha_{k+1}-\alpha_{k}\right)\right.$, that is of length $\omega^{\text {weight }(r)+1}\left(\alpha_{k+1}-\alpha_{k}\right)$. Hence $r^{\prime}=r_{1} r_{2} r_{3} \ldots$ is path, it is congruent to $r$ and of length $\omega^{\text {weight }(r)+1} \times \alpha$. It is worth observing that $B_{l i m}\left(r^{\prime}\right)=A$.

Because of the translation from formulae to automata, we can also establish a pumping lemma at the level of formulae.

\section{Lemma 3.5.}

(I): Let $\mathcal{A}$ be a simple ordinal automaton with acceptance conditions and $\alpha, \beta$ be countable ordinals such that $\alpha \approx_{\operatorname{card}(B)+2} \beta$. Then, $\mathcal{A}$ has an accepting run of length $\alpha$ iff $\mathcal{A}$ has an accepting run of length $\beta$.

(II): Let $\phi$ be a formula in $\operatorname{LTL}(\mathrm{U}, \mathrm{S})$ and $\alpha, \beta$ be countable ordinals such that $\alpha \approx_{|\phi|+2} \beta$. Then $\phi$ has an $\alpha$-model iff $\phi$ has a $\beta$-model.

Proof.

(I): Direct consequence of Lemma 3.2 and Lemma 3.4 since accepting runs can be viewed as paths.

(II): By Lemma 2.3, $\phi$ has an $\alpha$-model iff $\mathcal{A}_{\phi}$ has an accepting run $r$ of length $\alpha$. Since the cardinal of the basis of $\mathcal{A}_{\phi}$ is precisely $|\phi|$, by (I) we get that $\mathcal{A}_{\phi}$ has an accepting run $r$ of length $\alpha$ iff $\mathcal{A}_{\phi}$ has an accepting run $r$ of length $\beta$. Equivalently, $\phi$ has a $\beta$-model.

\section{Checking nonemptiness of Simple ordinal automata}

In this section, we provide algorithms to check whether a simple ordinal automaton admits accepting runs. The first one runs in exponential time. Our optimal algorithm runs in polynomial space in the size of the basis (see Section 4.2).

4.1. An exponential-time algorithm for checking nonemptiness. Let $\mathcal{A}$ be a simple ordinal automaton $\left\langle B, Q, I, F, \mathcal{F}, \delta_{\text {next }}, \delta_{\text {lim }}\right\rangle$. We provide below an algorithm to check given $q, q^{\prime} \in Q$ and $n \in \mathbb{N}$ whether there is path $r: \alpha+1 \rightarrow Q$ such that $r(0)=q, r(\alpha)=q^{\prime}$ and $\alpha<\omega^{n}$. Given an $(\alpha+1)$-path we write abs $(r)$ to denote the triple $\langle r(0)$, all $(r), r(\alpha)\rangle$. We define a family of relations containing the triples of the form $\operatorname{abs}(r)$. Each relation $R_{i}$ below is therefore a subset of $Q \times \mathcal{P}(B) \times Q$.

- $R_{0}=\left\{\left\langle q, q \cap q^{\prime}, q^{\prime}\right\rangle:\left\langle q, q^{\prime}\right\rangle \in \delta_{\text {next }}\right\}$,

- For $i \in \mathbb{N}$,

$$
R_{i}^{\prime}=\left\{\left\langle q_{0}, \bigcap_{j=0}^{m} A_{j}, q_{m+1}\right\rangle: \exists q_{0}, \ldots, q_{m+1}, A_{0}, \ldots, A_{m} \text { s.t. } \bigwedge_{j=0}^{m}\left\langle q_{j}, A_{j}, q_{j+1}\right\rangle \in R_{i}\right\}
$$

- For $i \in \mathbb{N}, R_{i+1}$ is defined from $R_{i}^{\prime}$ as follows: $\left\langle q, A, q^{\prime}\right\rangle \in R_{i+1}$ iff one of the conditions holds true:

(1.): $\left\langle q, A, q^{\prime}\right\rangle \in R_{i}^{\prime}$, 
(2.): there exist $\left\langle q, A^{\prime}, q^{\prime \prime}\right\rangle \in R_{i}^{\prime}(2.1),\left\langle q^{\prime \prime}, Y, q^{\prime \prime}\right\rangle \in R_{i}^{\prime}$ and a limit transition $\left\langle Y, q^{\prime}\right\rangle \in \delta_{\text {lim }}$ (2.2) such that $A=A^{\prime} \cap Y \cap q^{\prime}$.

The above numbering will be reused in Figure 2,

Let us first observe a few facts, whose proofs are by an easy verification.

(1) Whenever $\left\langle q, A, q^{\prime}\right\rangle \in R_{i}, A \subseteq q \cap q^{\prime}$.

(2) Because $R_{i} \subseteq R_{i+1}$ for all $i$, for some $N \leq 2^{3 \times \operatorname{card}(B)}+1, R_{N+1}=R_{N}$. The bound $2^{3 \times \operatorname{card}(B)}+1$ takes simply into account that $Q \subseteq \mathcal{P}(B)$.

In the sequel, for $n \geq 0$ and for $\left\langle q, A, q^{\prime}\right\rangle \in Q \times \mathcal{P}(B) \times Q$, we establish the equivalence of the propositions below:

- there is $\alpha+1<\omega^{n+1}$ and an $(\alpha+1)$-path $r$ such that $\operatorname{abs}(r)=\left\langle q, A, q^{\prime}\right\rangle$,

- $\left\langle q, A, q^{\prime}\right\rangle \in R_{n}^{\prime}$.

Lemma 4.1. If $\left\langle q, A, q^{\prime}\right\rangle \in R_{n}$, then there exist $\alpha<\omega^{n}$ and an $(\alpha+1)$-path such that $\operatorname{abs}(r)=\left\langle q, A, q^{\prime}\right\rangle$.

Proof. The proof is by induction on $n$. For the base $n=0$, the proof is by an easy verification. In the induction step, suppose that $\left\langle q, A, q^{\prime}\right\rangle \in R_{n+1}$. First suppose that $\left\langle q, A, q^{\prime}\right\rangle \in R_{n}^{\prime}$, that is there are $q_{0}, \ldots, q_{m+1}, A_{0}, \ldots, A_{m}$ such that $\bigwedge_{j=0}^{m}\left\langle q_{j}, A_{j}, q_{j+1}\right\rangle \in R_{n}$, $A=\bigcap_{j=0}^{m} A_{j}, q_{0}=q$ and $q_{m+1}=q^{\prime}$. By induction hypothesis, for $i \in[0, m]$, there is a path $r_{i}: \alpha_{i}+1 \rightarrow Q$ such that $\operatorname{abs}\left(r_{i}\right)=\left\langle q_{i}, A_{i}, q_{i+1}\right\rangle$ and $\alpha_{i}<\omega^{n}$. Hence, $r_{0} \cdots r_{m}$ is a path of the desired form of length strictly less than $\omega^{n}$.

If $\left\langle q, A, q^{\prime}\right\rangle \notin R_{n}^{\prime}$, then necessarily, by definition of $R_{n+1}$, there exist $\left\langle Y, q^{\prime}\right\rangle \in \delta_{\text {lim }}$, $\left\langle q, A^{\prime}, q^{\prime \prime}\right\rangle \in R_{n}^{\prime}$ and $\left\langle q^{\prime \prime}, Y, q^{\prime \prime}\right\rangle \in R_{n}^{\prime}$ such that $A=A^{\prime} \cap Y \cap q^{\prime}$.

Hence, by definition of $R_{n}^{\prime}$ and by induction hypothesis there is a path $r: \alpha+1 \rightarrow Q$ of length strictly less than $\omega^{n}$ between $q$ and $q^{\prime \prime}$. Similarly, there is a path $r^{\prime}: \beta+1 \rightarrow Q$ of length strictly less than $\omega^{n}$ between $q^{\prime \prime}$ and $q^{\prime \prime}$. Observe that $r^{\prime \prime}=r \cdot\left(r^{\prime}\right)^{\omega} q^{\prime}$ is a path of length strictly less than $\omega^{n+1}, B_{l i m}\left(r^{\prime \prime}\right)=Y$ and $\operatorname{abs}\left(r^{\prime \prime}\right)=\left\langle q, A, q^{\prime}\right\rangle$.

Consequently, if $\left\langle q, A, q^{\prime}\right\rangle \in R_{n}^{\prime}$, then there is $\alpha+1<\omega^{n+1}$ and an $(\alpha+1)$-path $r$ such that $\operatorname{abs}(r)=\left\langle q, A, q^{\prime}\right\rangle$. A converse result can also be established.

Lemma 4.2. Let $r: \alpha+1 \rightarrow Q$ be a path such that $\alpha<\omega^{n}$. Then $\operatorname{abs}(r) \in R_{n}^{\prime}$.

Proof. The proof is by induction on $n$. The base case $n=0$ is immediate. In the induction step, let $r$ be a path of length $\alpha<\omega^{n+1}$. If $\alpha<\omega^{n}$, by induction hypothesis $\langle r(0)$, all $(r), r(\alpha)\rangle \in R_{n}^{\prime}$ and therefore $\langle r(0)$, all $(r), r(\alpha)\rangle \in R_{n+1}$ since $R_{n}^{\prime} \subseteq R_{n+1}$. Now suppose that $\alpha=\omega^{n} \times m+\beta$ with $\beta<\omega^{n}$ and $m>0$. In order to show that $\langle r(0), \operatorname{all}(r), r(\alpha)\rangle \in$ $R_{n+1}^{\prime}$ it is sufficient to consider the case $\alpha=\omega^{n}$. Indeed, $R_{n+1}^{\prime}$ is closed under composition, i.e. if $\left\langle q_{0}, A_{0}, q_{0}^{\prime}\right\rangle \in R_{n+1}^{\prime}$ and $\left\langle q_{0}^{\prime}, A_{1}, q_{1}^{\prime}\right\rangle \in R_{n+1}^{\prime}$, then $\left\langle q_{0}, A_{0} \cap A_{1}, q_{1}^{\prime}\right\rangle \in R_{n+1}^{\prime}$. So, suppose that $r$ is of length $\omega^{n}+1$. By induction hypothesis, for every $0 \leq i<i^{\prime}$, $\left\langle r\left(\omega^{n-1} \times i\right), A_{i, i^{\prime}}, r\left(\omega^{n-1} \times i^{\prime}\right)\right\rangle \in R_{n}^{\prime}$ for some $A_{i, i^{\prime}}$. By Ramsey's Theorem, there are $0<i_{0}<i_{1}<\ldots$ such that $\left\langle r\left(\omega^{n-1} \times i_{k}\right), A_{i_{k}, i_{k+1}}, r\left(\omega^{n-1} \times i_{k+1}\right)\right\rangle$ is the same for all $k \geq 0$. Let $j=i_{0}$ and $j^{\prime}=i_{1}$. By induction hypothesis, $\left\langle r\left(\omega^{n-1} \times j\right), A_{j, j^{\prime}}, r\left(\omega^{n-1} \times j^{\prime}\right)\right\rangle \in R_{n}^{\prime}$ since the length of $r_{\left[\omega^{n-1} \times j, \omega^{n-1} \times j^{\prime}\right]}$ is strictly less than $\omega^{n}$. Moreover, we have $A_{j, j^{\prime}}=B_{l i m}\left(r, \omega^{n}\right)$. So, there exist $\left\langle r(0), A^{\prime}, q^{\prime \prime}\right\rangle \in R_{n}^{\prime}\left(A^{\prime}=A_{0, j}, q^{\prime \prime}=r\left(\omega^{n-1} \times j\right)\right),\left\langle q^{\prime \prime}, Y, q^{\prime \prime}\right\rangle \in R_{n}^{\prime}\left(Y=A_{j, j^{\prime}}\right)$ and a limit transition $\left\langle Y, r\left(\omega^{n}\right)\right\rangle \in \delta_{\text {lim }}$ such that $A=A^{\prime} \cap Y \cap r\left(\omega^{n}\right)$. Consequently, $\langle r(0), \operatorname{all}(r), r(\alpha)\rangle \in R_{n+1}^{\prime}$. 
We provide below a first complexity result.

Lemma 4.3. The nonemptiness problem for simple ordinal automata with acceptance conditions can be checked in exponential time in $\operatorname{card}(B)$.

Proof. Let $\mathcal{A}$ be of the form $\left\langle B, Q, I, F, \mathcal{F}, \delta_{\text {next }}, \delta_{\text {lim }}\right\rangle . \mathcal{A}$ has an accepting run iff either (A) there are $q_{0} \in I, q_{f} \in F$ and $A \subseteq B$ such that $\left\langle q_{0}, A, q_{f}\right\rangle \in R_{n}^{\prime}$ for some $n$ or (B) there are $q_{0} \in I$, and a run $r$ from $q_{0}$ such that $B_{\text {lim }}(r) \in \mathcal{F}$. (A) deals with accepting runs of length some successor ordinal, whereas $(\mathbf{B})$ deals with accepting runs of length some limit ordinal.

In order to check $(\mathbf{A})$, it is sufficient to test for $\left\langle q_{0}, A, q_{f}\right\rangle \in I \times \mathcal{P}(B) \times F$ whether $\left\langle q_{0}, A, q_{f}\right\rangle \in R_{\text {card }(B)+3}^{\prime} \subseteq R_{\text {card }(B)+4}$. Since $\operatorname{card}(Q)$ is in $\mathcal{O}\left(2^{\text {card }(B)}\right)$, computing $R_{\text {card }(B)+4}$ takes card $(B)+4$ steps that requires polynomial time in $|\mathcal{A}|$ and exponential time in $\operatorname{card}(B)$, we obtain the desired result. Observe that we can take advantage of the fact that computing the transitive closure of a relation and the maximal strongly connected components can be done in polynomial time in the size of the relations.

By Ramsey's theorem, $(\mathbf{B})$ is equivalent to the following condition: there are $q \in Q$, $A \subseteq B, A^{\prime} \in \mathcal{F}$ and runs $r_{1}$ and $r_{2}$ such that $\operatorname{abs}\left(r_{1}\right)=\left\langle q_{0}, A, q\right\rangle$ and $\operatorname{abs}\left(r_{2}\right)=\left\langle q, A^{\prime}, q\right\rangle$.

Hence. in order to check these, it is enough to check whether there are $q_{0} \in I, q \in Q$ and $A \subseteq B$ such that $\left\langle q_{0}, A, q\right\rangle \in R_{\text {card }(B)+3}^{\prime},\left\langle q, A^{\prime}, q\right\rangle \in R_{\text {card }(B)+3}^{\prime}$ and $A^{\prime} \in \mathcal{F}$. This can be done in exponential time as for $(\mathbf{A})$.

As a corollary of Lemma 4.3, satisfiability for $\operatorname{LTL}(\mathrm{U}, \mathrm{S})$ is in EXPTIME. Moreover, this can be improved as shown in the proof of Theorem 5.1 presented in Section 5 .

4.2. A polynomial-space algorithm. We improve below the bound in Lemma 4.3 by taking advantage that the recursive depth is linear and only paths of at most exponential length need to be computed.

Theorem 4.4. The nonemptiness problem for simple ordinal automata can be checked in polynomial space in $\operatorname{card}(B)$.

Proof. By Lemma 4.1 and Lemma 4.2 and by the fact that for all $n \geq 0$, we have $R_{n} \subseteq$ $R_{\text {card }(B)+4}$, we obtain that $\mathcal{A}$ has an accepting run iff $(\mathbf{A})$ there are $q_{0} \in I, q_{f} \in F$ and $A \subseteq B$ such that $\left\langle q_{0}, A, q_{f}\right\rangle \in R_{\mathrm{card}(B)+3}$ or $(\mathbf{B})$ there are $q_{0} \in I, q \in Q$ and $A^{\prime} \subseteq B$ such that $\left\langle q_{0}, A^{\prime}, q\right\rangle \in R_{\operatorname{card}(B)+4},\left\langle q, A^{\prime}, q\right\rangle \in R_{\text {card }(B)+4}$ and $A^{\prime} \in \mathcal{F}$. $B$ denotes the basis of $\mathcal{A}$.

The function PATH defined in Figure 2 checks recursively whether a triple belongs to $R_{N}$. Typically, the specification is that there exists an accepting computation for $\operatorname{PATH}\left(\mathcal{A},\left\langle q, A, q^{\prime}\right\rangle, N\right)$ iff $\left\langle q, A, q^{\prime}\right\rangle \in R_{N}$ for the ordinal automaton $\mathcal{A}$. It takes into account that the number of potential triples in $R_{N}$ is bounded. Observe that the algorithm is nondeterministic and any guess that breaks some condition somewhere aborts the computation.

In order to check $(\mathbf{A})$, the non-deterministic algorithm guesses $q_{0} \in I, q_{f} \in F$ and $A \subseteq B$ (encoded in polynomial space in $\mathcal{O}(\operatorname{card}(B))$ and test whether

$$
\operatorname{PATH}\left(\mathcal{A},\left\langle q_{0}, A, q_{f}\right\rangle, \operatorname{card}(B)+4\right)
$$

returns true. Condition (B) admits a similar treatment. The non-deterministic algorithm PATH defined below works in polynomial space in $\operatorname{card}(B)$ assuming that the last argument is polynomial in $\operatorname{card}(B)$ which is the case with $\operatorname{card}(B)+4$. 


\section{$\operatorname{PATH}\left(\mathcal{A},\left\langle q, A, q^{\prime}\right\rangle, N\right)$}

- If $N=0$ then (if (either $A \neq q \cap q^{\prime}$ or $\left\langle q, q^{\prime}\right\rangle \notin \delta_{\text {next }}$ ) then abort else return true);

- If $N>0$ then go non-deterministically to 1 . or 2 .

(1.): Guess on-the-fly a sequence

$$
\left\langle q_{0}, A_{0}, q_{1}\right\rangle,\left\langle q_{1}, A_{1}, q_{2}\right\rangle, \ldots,\left\langle q_{m}, A_{m}, q_{m+1}\right\rangle
$$

such that

$$
\begin{aligned}
& -m<2^{3 \times \operatorname{card}(B)+1}+1 \\
& - \text { for } 0 \leq i \leq m, \operatorname{PATH}\left(\mathcal{A},\left\langle q_{i}, A_{i}, q_{i+1}\right\rangle, N-1\right) \text { returns true, } \\
& -A=\bigcap_{j} A_{j} \\
& -q=q_{0}, q^{\prime}=q_{m+1}
\end{aligned}
$$

(2.): We guess here two long sequences:

(2.1): Guess on-the-fly a sequence

$$
\left\langle q_{0}, A_{0}, q_{1}\right\rangle,\left\langle q_{1}, A_{1}, q_{2}\right\rangle, \ldots,\left\langle q_{m}, A_{m}, q_{m+1}\right\rangle
$$

such that

$$
\begin{aligned}
& \text { - } m<2^{3 \times \operatorname{card}(B)+1}+1, \\
& \text { - for } 0 \leq i \leq m, \operatorname{PATH}\left(\mathcal{A},\left\langle q_{i}, A_{i}, q_{i+1}\right\rangle, N-1\right) \text { returns true, } \\
& \text { - } A^{\prime}=\bigcap_{j} A_{j} ; \\
& \text { - } q_{0}=q ;
\end{aligned}
$$

(2.2): Guess a limit transition $\left\langle Y, q^{\prime}\right\rangle \in \delta_{\text {lim }}$ and on-the-fly a sequence

$$
\left\langle q_{0}^{\prime}, A_{0}^{\prime}, q_{1}^{\prime}\right\rangle,\left\langle q_{1}^{\prime}, A_{1}^{\prime}, q_{2}^{\prime}\right\rangle, \ldots,\left\langle q_{m}^{\prime}, A_{m^{\prime}}^{\prime}, q_{m^{\prime}+1}^{\prime}\right\rangle
$$

such that

$$
\begin{aligned}
& \quad-m^{\prime}<2^{3 \times \operatorname{card}(B)+1}, \\
& \quad-\text { for } 0 \leq i \leq m^{\prime}, \operatorname{PATH}\left(\mathcal{A},\left\langle q_{i}^{\prime}, A_{i}^{\prime}, q_{i+1}^{\prime}\right\rangle, N-1\right) \text { returns true, } \\
& -A=\left(A^{\prime} \cap q_{m^{\prime}+1}^{\prime}\right) \cap \bigcap_{j} A_{j}^{\prime}, Y=\bigcap_{j} A_{j}^{\prime}, \\
& -q_{0}^{\prime}=q_{m+1} ;
\end{aligned}
$$

Figure 2: Algorithm PATH

In (1.), guessing on-the-fly a long sequence means that only two consecutive triples are kept in memory at any time. We introduce a counter that will guarantee that $m<2^{3 \times \operatorname{card}(B)+1}$ and it requires only space in $\mathcal{O}(\operatorname{card}(B))$. Moreover, in order to check $A=\bigcap_{j} A_{j}$ we need two auxiliary variables that bookkeep the $A_{j}$ computed so far. Similar techniques are used in (2.) to guarantee that this non-deterministic algorithm requires only polynomial space in $\mathcal{O}(\operatorname{card}(B)+N)$ (we only need more variables and steps). It is straightforward to show that $\operatorname{PATH}\left(\mathcal{A},\left\langle q, A, q^{\prime}\right\rangle, N\right)$ has a computation that returns true (all the guesses were correct) iff $\left\langle q, A, q^{\prime}\right\rangle \in R_{N}$. Finally, by using Savitch Theorem [Sav70], we can conclude that nonemptiness can be checked in deterministic polynomial space in $\operatorname{card}(B)$.

Observe that the algorithm in the proof of Theorem 4.4 runs in space $\mathcal{O}(\operatorname{card}(B) \times$ $\left(\operatorname{card}(B)+\log (\operatorname{card}(Q))+\log \left(\operatorname{card}\left(\delta_{\text {lim }}\right)\right)+\log \left(\operatorname{card}\left(\delta_{\text {next }}\right)\right)\right)$. Indeed, the recursive depth is in $\mathcal{O}(\operatorname{card}(B))$. This is certainly sufficient to get forthcoming results about the complexity of $\operatorname{LTL}(\mathrm{U}, \mathrm{S})$. Nevertheless, the exact complexity characterization of the nonemptiness problem is open. It seems unlikely that the problem can be solved in NLOGSPACE. 


\section{Complexity of Satisfiability Problems}

We establish new complexity results for problems related to LTL(U,S) satisfiability which follow from the intermediate results we have established so far.

5.1. Complexity of $\operatorname{LTL}(\mathrm{U}, \mathrm{S})$. Here is the main result of the paper.

Theorem 5.1. The satisfiability problem for $\operatorname{LTL}(\mathrm{U}, \mathrm{S})$ over the class of ordinals is PSPACEcomplete.

Proof. By Theorem 1.2(II), a formula is satisfiable iff it is satisfiable on some model of countable length. By Lemma 2.3, given a formula $\phi$ in $\operatorname{LTL}(\mathrm{U}, \mathrm{S})$, there is an automaton $\mathcal{A}_{\phi}$ whose accepting runs correspond exactly to models of $\phi$. In order to check nonemptiness of $\mathcal{A}_{\phi}$, we do not build it explicitly (as usual) but we run the algorithm from the proof of Theorem 4.4 and we compute the locations, and transition relations of $\mathcal{A}_{\phi}$ on demand. Hence, we obtain a polynomial space non-deterministic algorithm since the cardinality of the basis of $\mathcal{A}_{\phi}$ is in $\mathcal{O}(|\phi|)$ and checking whether a subset of $B$ is a location of $\mathcal{A}_{\phi}$ or $\left\langle q, q^{\prime}\right\rangle \in \delta_{\text {next }}$ or $\langle Y, q\rangle \in \delta_{\text {lim }}$ can be done in polynomial space in $\mathcal{O}(|\phi|)$. Again by Savitch Theorem [Sav70], we get that the satisfiability problem for LTL $(U, S)$ is in PSPACE. The PSPACE lower bound can be easily shown inherited from LTL.

Our procedure to show the PSPACE upper bound is not optimal and it is subject to many refinements but it is sufficient for our needs. For instance, it is possible to have as a base set for $\mathcal{A}_{\phi}$ the subset of $\operatorname{sub}(\phi)$ made of until or since subformulae and propositional variables. Indeed, the implicit presence of other subformulae can be deduced thanks to maximal consistency. This refinement possibly decreases the length of the small models.

Due to Kamp's Theorem [Kam68], we get the following corollary.

Corollary 5.2. Let $\operatorname{LTL}\left(\mathrm{U}, \mathrm{S}, \mathrm{O}_{1}, \ldots, \mathrm{O}_{k}\right)$ be an extension of $\operatorname{LTL}(\mathrm{U}, \mathrm{S})$ with $k$ first-order definable temporal operators.

Then the satisfiability problem for the logic $\operatorname{LTL}\left(\mathrm{U}, \mathrm{S}, \mathrm{O}_{1}, \ldots, \mathrm{O}_{k}\right)$ over the class of ordinals is in PSPACE.

Indeed, every formula $\mathrm{O}_{i}\left(p_{1}, \ldots, p_{n_{i}}\right)$ encoded as a DAG can be translated into an equivalent formula in $\operatorname{LTL}(\mathrm{U}, \mathrm{S})$ encoded as a DAG over the propositional variables $p_{1}, \ldots, p_{n_{i}}$. Since $\mathrm{O}_{1}, \ldots, \mathrm{O}_{k}$ and their definition in $\operatorname{LTL}(\mathrm{U}, \mathrm{S})$ are constants of $\operatorname{LTL}\left(\mathrm{U}, \mathrm{S}, \mathrm{O}_{1}, \ldots, \mathrm{O}_{k}\right)$, we obtain a translation in polynomial-time (with our definition for the size of formulae).

5.2. A family of satisfiability problems. The satisfiability problem for LTL(U,S) asks for the existence of a model for a given formula. A natural variant of this problem consists in fixing the length of the models in advance as for LTL. The satisfiability problem for $\operatorname{LTL}(\mathrm{U}, \mathrm{S})$ over $\alpha$-models, noted $\operatorname{SAT}(\alpha, \operatorname{LTL}(\mathrm{U}, \mathrm{S}))$, is defined as follows:

input:: a formula $\phi$ in $\operatorname{LTL}(\mathrm{U}, \mathrm{S})$;

question:: Is $\phi$ satisfiable over an $\alpha$-model?

In this subsection we prove that $\operatorname{SAT}(\alpha, \operatorname{LTL}(\mathrm{U}, \mathrm{S}))$ is in PSPACE for every countable ordinal $\alpha$. First we consider the case of ordinals strictly less than $\omega^{\omega}$. Let us establish that for every $\alpha<\omega^{\omega}$ there is a formula $\operatorname{def}_{\alpha}$ in $\operatorname{LTL}(\mathrm{U}, \mathrm{S})$ with the truth constant $\top$ (no propositional variable) such that for every $\beta$-model $\sigma$, we have $\sigma, 0 \models \operatorname{def}_{\alpha}$ iff $\beta=\alpha$. 
Lemma 5.3. Given an ordinal $0<\alpha=\omega^{k_{1}} a_{k_{1}}+\cdots \omega^{k_{m}} a_{k_{m}}<\omega^{\omega}$ with $k_{1}>\ldots>k_{m} \geq 0$ and $a_{k_{1}}, \ldots a_{k_{m}} \in \mathbb{N} \backslash\{0\}$, there is a formula $\operatorname{def}_{\alpha}$ in $\operatorname{LTL}(\mathrm{U}, \mathrm{S})$ of linear size in $\sum_{i}\left(k_{i} \times a_{k_{i}}\right)$ such that for any model $\sigma$, we have $\sigma, 0 \models \operatorname{def}_{\alpha}$ iff $\sigma$ is of length $\alpha$.

Proof. We define a family $\left(\varphi_{i}\right)_{i \geq 0}$ such that for all $\alpha$-models $\sigma$ and $\beta<\alpha$, we have $\sigma, \beta \models \varphi_{i}$ iff $\beta$ is a multiple of $\omega^{i}$. We set $\varphi_{0}=\top$ and by induction $\varphi_{i+1}=\varphi_{i} \wedge \neg\left(\neg \varphi_{i} S \varphi_{i}\right)$. Observe that $\left|\varphi_{i}\right|$ is polynomial in $i$ since it is defined as the cardinality of $\operatorname{sub}\left(\varphi_{i}\right)$. Now let us define $\operatorname{def}_{\alpha}$ as $t(\alpha)$ defined recursively below:

- $t(1)=\neg \mathrm{F}^{+} \top, t(n)=\mathrm{X} t(n-1)$ for $n>1$,

- $t\left(\omega^{k_{1}} a_{k_{1}}+\cdots+\omega^{k_{m}} a_{k_{m}}\right)=\neg \varphi_{k_{1}} \mathrm{U}\left(\varphi_{k_{1}} \wedge t\left(\omega^{k_{1}}\left(a_{k_{1}}-1\right)+\cdots+\omega^{k_{m}} a_{k_{m}}\right)\right)$ with $k_{1}>0$ and $\left(a_{k_{1}} \geq 2\right.$ or $\left.m>1\right)$

- $t(\omega)=\mathrm{G}^{+} \mathrm{X}^{-1} \top \wedge \mathrm{F}^{+} \top \wedge \mathrm{G}^{+} \mathrm{X} \top$,

- $t\left(\omega^{k_{1}}\right)=\mathrm{G}^{+} \neg \varphi_{k_{1}} \wedge \mathrm{GF}^{+} \varphi_{k_{1}-1}$ with $k_{1}>1$.

The size of $\operatorname{def}_{\alpha}$ is in $\mathcal{O}\left(\sum_{i}\left(k_{i} \times a_{k_{i}}\right)\right)$.

We are now in position to state the following result.

Corollary 5.4. For every $\alpha<\omega^{\omega}$, the problem $\operatorname{SAT}(\alpha, \operatorname{LTL}(\mathrm{U}, \mathrm{S}))$ is in PSPACE.

Proof. $\phi$ has a $\alpha$-model iff $\psi=\phi \wedge \operatorname{def}_{\alpha}$ is satisfiable over the class of ordinals. Thanks to Lemma 5.3 and Theorem [5.1, we obtain the PSPACE upper bound.

Now we consider the case of a countable ordinal $\alpha \geq \omega^{\omega}$. Let $\alpha^{\prime}$ be the unique ordinal strictly less than $\omega^{\omega}$ such that $\alpha=\omega^{\omega} \times \gamma+\alpha^{\prime}$ for some ordinal $\gamma$. Note that for every $k, \operatorname{trunc}_{k}(\alpha)=\operatorname{trunc}_{k}\left(\omega^{k}+\alpha^{\prime}\right)<\omega^{\omega}$. By Lemma 3.5(II), $\phi$ has an $\alpha$-model iff $\phi$ has a $\alpha_{|\phi|}$-model with $\alpha_{|\phi|}=\operatorname{trunc}_{|\phi|+2}(\alpha)=\operatorname{trunc}_{|\phi|+2}\left(\omega^{|\phi|+2}+\alpha^{\prime}\right)$. Hence, $\phi$ has an $\alpha$-model iff $\phi \wedge \operatorname{def}_{\alpha_{|\phi|}}$ is satisfiable (over the class of countable ordinals). Since the size of $\operatorname{def}_{\alpha_{|\phi|}}$ is polynomial in the size of $\phi$, we derive from Theorem 5.1 the following result.

Corollary 5.5. For every countable $\alpha \geq \omega^{\omega}$, the problem $\operatorname{SAT}(\alpha, \operatorname{LTL}(\mathrm{U}, \mathrm{S}))$ is in PSPACE.

Corollaries 5.4, 5.5 and the arguments similar to the arguments in the proof of Corollary 5.2 imply the result below.

Theorem 5.6. The satisfiability problem for $\operatorname{LTL}\left(\mathrm{O}_{1}, \ldots, \mathrm{O}_{k}\right)$ restricted to $\alpha$-models is in PSPACE, for every finite set $\left\{\mathrm{O}_{1}, \ldots, \mathrm{O}_{k}\right\}$ of first-order definable temporal operators and for every countable ordinal $\alpha$.

Observe that $\alpha$ finite implies $\operatorname{SAT}\left(\alpha, \operatorname{LTL}\left(\mathrm{O}_{1}, \ldots, \mathrm{O}_{k}\right)\right)$ is NP-complete, otherwise PSPACE-I hardness for $\operatorname{SAT}(\alpha, \operatorname{LTL}(\mathrm{U}, \mathrm{S}))$ follows from $\operatorname{PSPACE}-\operatorname{completeness}$ of $\operatorname{SAT}(\omega, \operatorname{LTL}(\mathrm{U}, \mathrm{S}))$.

5.3. Uniform satisfiability. Büchi (see, e.g., [BS73]) has shown that there is a finite amount of data concerning any countable ordinal that determines its monadic theory.

Definition 5.7 (Code of an ordinal). Let $\alpha$ be a countable ordinal and let $m$ be in $[1, \omega]$.

(1) Write $\alpha=\omega^{m} \alpha^{\prime}+\zeta$ with $\zeta<\omega^{m}$ (this can be done in a unique way), and let

$$
p_{m}(\alpha):=\left\{\begin{array}{ll}
-2 & \text { if } \alpha^{\prime}=0 \\
-1 & \text { if } 0<\alpha^{\prime}<\omega_{1}
\end{array} .\right.
$$

(2) If $\zeta \neq 0$, write $\zeta=\sum_{i \leq n} \omega^{n-i} \cdot a_{n-i}$ where $a_{i} \in \omega$ for $i \leq n$ and $a_{n} \neq 0$ (this can be done in a unique way), and let $t_{m}(\alpha):=\left(a_{n}, \ldots, a_{0}\right)$. If $\zeta=0$, let $t_{m}(\alpha)=-3$. 
(3) The $m$-code of $\alpha$ is the pair $\left(p_{m}(\alpha), t_{m}(\alpha)\right)$.

The following is implicit in BS73.

Theorem 5.8 (Code Theorem). There is an algorithm that, given a monadic second-order sentence $\phi$ and the $\omega$-code of a countable ordinal $\alpha$, determines whether $\langle\alpha,<\rangle \models \phi$.

Lemma 3.5 can be rephrased as "the $(|\phi|+2)$-code of an ordinal $\alpha$ determines whether $\phi$ has a model of length $\alpha$ ".

Let $C=\left(b, a_{n}, \ldots a_{0}\right)$ be an $m$-code. Its size is defined as $n+a_{0}+a_{1}+\cdots+a_{n}$. It is clear that for $m_{1}<m_{2}$ the $m_{2}$-code of an ordinal determines its $m_{1}$-code and there is a linear-time algorithm, that given $m_{2}$-code of an ordinal and $m_{1}<m_{2}$ computes the $m_{1}$-code of the ordinal.

The arguments used in the proof of Corollary 5.5 show the following theorem.

Theorem 5.9 (Uniform Satisfiability).

(I): There is a polynomial-space algorithm that, given an LTL(U,S) formula $\phi$ and the $\omega$-code of a countable ordinal $\alpha$, determines whether $\phi$ has an $\alpha$-model.

(II): There is a polynomial-space algorithm that, given an LTL(U,S) formula $\phi$ and the $(|\phi|+2)$-code of a countable ordinal $\alpha$, determines whether $\phi$ has an $\alpha$-model.

\section{Related Work}

In this section, we compare our results with those from the literature related to satisfiability. It is worth noting that an axiomatization of $\operatorname{LTL}(\mathrm{U}, \mathrm{S})$ over ordinals can be found in [Ven93. Nevertheless, the concern in this above-mentioned paper is quite different from ours.

6.1. Comparison with Rohde's thesis. In Roh97, it is shown that an uniform satisfiability problem for temporal logic with until (and without since) can be solved in exponential time (flows of time are countable ordinals). The inputs of this problem are a formula in $\operatorname{LTL}(\mathrm{U})$ and the representation of a countable ordinal. The satisfiability problem is also shown in ExPTIME. In order to obtain this upper bound, formulae are shown equivalent to alternating automata and a reduction from alternating automata into a specific subclass of non-deterministic automata is given. Finally, a procedure for testing nonemptiness is provided. Here are the similarities between [Roh97] and our results.

(1) We also follow an automata-based approach and the class of non-deterministic automata in [Roh97] and ours have a structured set of locations and limit transitions use elements that are true from some position.

(2) Existence of $\alpha$-paths in the automata depends on some truncation of $\alpha$.

(3) The logical decision problems can be solved in exponential time.

However, our work improves some results from [Roh97.

(1) Our temporal logic includes the until and since operators (instead of until only) and it is therefore as expressive as first-order logic.

(2) We establish a tight PSPACE upper bound (instead of EXPTIME) thanks to the introduction of simple ordinal automata.

(3) Our proofs are shorter and more transparent (instead of the lengthy developments found in [Roh97]). 
Consequently, the developments from [Roh97] and ours follow the same approach with different definitions for automata, different intermediate lemmas and distinct final complexity bounds. On the other hand, the structure of the whole proof to obtain the main complexity bounds is similar.

6.2. LTL over other classes of linear orderings. Even though the results for lineartime temporal logics from Rey03, Rey10b involve distinct models, our automata-based approach has similarities with these works that uses a different proof method, namely mosaics. Indeed, equivalence classes of the relation $\sim$ between runs of length a successor ordinal roughly correspond to mosaics from Rey03. We recall the main results below.

\section{Theorem 6.1.}

(I): Rey10b The satisfiability problem for the temporal logic with until and since over the reals is PSPACE-complete.

(II): Rey03 The satisfiability problem for LTL(U) over the class of all linear orders is PSPACE-complete.

The proofs in Rey03, Rey10b are much more involved than our proofs since the orders are more complex than the class of ordinals. Moreover, a recent work [Cri09] has established that $\operatorname{LTL}(\mathrm{U}, \mathrm{S})$ over the class of linear orderings has an elementary complexity by using transducers as done in [Mic84] for standard LTL. More precisely, satisfiability for LTL(U,S) augmented with future and past Stavi operators is in 2Expspace Cri09. Nevertheless, complexity of LTL(U,S) over the class of linear orderings has been recently solved: for any temporal logic with a finite set of modalities definable in the existential fragment of secondorder logic has a PSPACE satisfiability problem over the class of linear orderings Rab10a, Rab10b. (see also Rey10a). Moreover, observe that LTL(U,S) over the reals has been recently shown in PSPACE in Rey10a, which allows us to obtain in a different way that $\mathrm{LTL}(\mathrm{U}, \mathrm{S})$ over the countable ordinals is in PSPACE (see the full arguments in Rab10a, Section 13]).

6.3. Quantitative temporal operators. In this section, we show that the main results from [DN07] are subsumed by the current paper. We also solve an open problem from Cac06, DN07. For every fixed countable ordinal $\alpha \leq \omega$, let us introduce the logic $\operatorname{LTL}\left(\mathcal{O}_{\alpha}\right)$ where the set of temporal operators $\mathcal{O}_{\alpha}$ is defined as follows: $\left\{\mathrm{X}^{\beta}: \beta<\omega^{\alpha}\right\} \cup\left\{\mathrm{U}^{\beta}\right.$ : $\left.\beta \leq \omega^{\alpha}\right\}$. The models of $\operatorname{LTL}\left(\mathcal{O}_{\alpha}\right)$ as those of $\operatorname{LTL}(\mathrm{U}, \mathrm{S})$ and the formulae of $\operatorname{LTL}\left(\mathcal{O}_{\alpha}\right)$ are precisely defined by:

$$
\phi::=p|\neg \phi| \phi_{1} \wedge \phi_{2}\left|\mathrm{X}^{\beta} \phi\right| \phi_{1} \mathrm{U}^{\beta} \phi_{2} .
$$

The satisfaction relation is inductively defined below where $\sigma$ is a model for $\operatorname{LTL}\left(\mathcal{O}_{\alpha}\right)$ (we omit the obvious clauses):

- $\sigma, \beta \models \mathrm{X}^{\beta^{\prime}} \phi$ iff $\beta+\beta^{\prime}$ is a position of $\sigma$ and $\sigma, \beta+\beta^{\prime} \models \phi$,

- $\sigma, \beta \models \phi_{1} \mathrm{U}^{\beta^{\prime}} \phi_{2}$ iff there is $\gamma \in\left(0, \beta^{\prime}\right)$ such that $\beta+\gamma$ is a position of $\sigma$, we have $\sigma, \beta+\gamma \models \phi_{2}$ and for every $\gamma^{\prime} \in(0, \gamma)$, we have $\sigma, \beta+\gamma^{\prime} \models \phi_{1}$.

The satisfiability problem for $\operatorname{LTL}\left(\mathcal{O}_{\alpha}\right)$ consists in determining, given a formula $\phi$, whether there is a model $\sigma$ such that $\sigma, 0 \models \phi$. The main results of [Cac06, DN07] are the following: 
(1) For every $k \in \mathbb{N} \backslash\{0\}$, the satisfiability problem for $\operatorname{LTL}\left(\mathcal{O}_{k}\right)$ restricted to models of length $\omega^{k}$ is PSPACE-complete when the natural numbers occurring in formulae are encoded in unary. With binary representation, it becomes EXPSPACE-hard (mainly because a temporal operator $\mathrm{X}^{2^{n}}$ is helpful to specify concisely the cell contents of exponential-space Turing machines).

(2) $\operatorname{LTL}\left(\mathcal{O}_{\omega}\right)$ restricted to models of length $\omega^{\omega}$ is decidable.

Observe that $\operatorname{LTL}\left(\mathcal{O}_{k}\right)$ cannot express the temporal operator $\mathrm{U}$ over the class of countable ordinals but it can do it on models of length $\omega^{k}$. Hence, each logic $\operatorname{LTL}\left(\mathcal{O}_{k}\right)$ is less expressive than $\operatorname{LTL}(\mathrm{U}, \mathrm{S})$.

Moreover, it is easy to show that for every $\alpha \leq \omega$, the logic $\operatorname{LTL}\left(\mathcal{O}_{\alpha}\right)$ is expressively equivalent (over the class of countable ordinals) to its sublogic over the following set $\mathcal{O}_{\alpha}^{\prime}$ of temporal operators:

$$
\mathcal{O}_{\alpha}^{\prime}=\left\{\mathbf{X}^{\omega^{i}}: \omega^{i}<\omega^{\alpha}, i \in \mathbb{N}\right\} \cup\left\{\mathrm{U}^{\omega^{\beta}}: \omega^{\beta} \leq \omega^{\alpha}, \beta \leq \omega\right\} .
$$

This set is finite when $\alpha$ is finite. Moreover, there is a linear-time (and logarithmic space) meaning preserving translation from $\operatorname{LTL}\left(\mathcal{O}_{\alpha}\right)$ into $\operatorname{LTL}\left(\mathcal{O}_{\alpha}^{\prime}\right)$.

Let us translate $\phi$ in $\operatorname{LTL}\left(\mathcal{O}_{\omega}^{\prime}\right)$ into a formula $t(\phi)$ in $\operatorname{LTL}(\mathrm{U}, \mathrm{S})$ homomorphically for the Boolean operators and such that the propositional variables remain unchanged. Here are the remaining clauses of translation:

- $t\left(\psi_{1} \mathrm{U}^{\omega^{i}} \psi_{2}\right)=\left(\neg \varphi_{i} \wedge t\left(\psi_{1}\right)\right) \mathrm{U}\left(\neg \varphi_{i} \wedge t\left(\psi_{2}\right)\right), t\left(\psi_{1} \mathrm{U}^{\omega^{\omega}} \psi_{2}\right)=t\left(\psi_{1}\right) \mathrm{U} t\left(\psi_{2}\right)$,

- $t\left(\mathrm{X}^{\omega^{i}} \psi_{1}\right)=\neg \varphi_{i} \mathrm{U}\left(\varphi_{i} \wedge t\left(\psi_{1}\right)\right)$.

The formula $\varphi_{i}$ is defined in the proof of Lemma 5.3. The following result is easy to show.

\section{Lemma 6.2.}

(I): Let $\phi$ be in $\operatorname{LTL}\left(\mathcal{O}_{\omega}^{\prime} \backslash\left\{\mathrm{U}^{\omega^{\omega}}\right\}\right) . t(\phi)$ is equivalent to $\phi$ over the class of countable ordinals, i.e. for all $\alpha$-models $\sigma$ and $\beta<\alpha$, we have $\sigma, \beta \models \phi$ iff $\sigma, \beta \models t(\phi)$.

(II): Let $\phi$ be in $\operatorname{LTL}\left(\mathcal{O}_{\omega}^{\prime}\right)$. For all $\omega^{\omega}$-models $\sigma$ and $\beta<\omega^{\omega}$, we have $\sigma, \beta \models \phi$ iff $\sigma, \beta \models t(\phi)$. Moreover, $|t(\phi)|$ is linear in $|\phi|$.

(I) is essentially based on the properties of formulae $\varphi_{i}$ and on the exclusion of $\mathrm{U}^{\omega^{\omega}}$. (II) simply takes advantage of the fact that for the $\omega^{\omega}$-models, $U$ and $U^{\omega^{\omega}}$ are obviously equivalent.

We obtain alternative proofs for known results and we get new results.

Theorem 6.3. For every $k \in \mathbb{N} \backslash\{0\}$,

(I): the satisfiability problem for $\operatorname{LTL}\left(\mathcal{O}_{k}\right)$ over $\omega^{k}$-models is in PSPACE with unary encoding of natural numbers,

(II): the satisfiability problem for $\operatorname{LTL}\left(\mathcal{O}_{k}^{\prime}\right)$ restricted to $\omega^{k}$-models is PSPACE-complete,

(III): for every countable infinite ordinal $\alpha$, the satisfiability problem for $\operatorname{LTL}\left(\mathcal{O}_{k}^{\prime}\right)$ restricted to $\alpha$-models is PSPACE-complete.

(III) is an instance of Theorem 5.6. (II) is an instance of (III) (with unary encoding of natural numbers). (I) can be shown by observing that there is a logarithmic space meaning preserving translation from $\operatorname{LTL}\left(\mathcal{O}_{k}\right)$ to $\operatorname{LTL}\left(\mathcal{O}_{k}^{\prime}\right)$. (I) is the main result of [DN07] with the unary encoding of natural numbers occurring in ordinal expressions. Finally, the corollary below improves the non-elementary bounds obtained in [Cac06, DN07] for $\operatorname{LTL}\left(\mathcal{O}_{\omega}\right)$ by reducing this temporal logic to the monadic second order logics, and then to Buchi ordinal automata. 
Corollary 6.4. Satisfiability for $\operatorname{LTL}\left(\mathcal{O}_{\omega}\right)$ over the class of $\omega^{\omega}$-models is PSPACE-complete with unary encoding of natural numbers in formulae.

\section{Conclusion}

In the paper, we have shown that the linear-time temporal logic with until and since over the class of ordinals, namely LTL(U,S) has a PSPACE-complete satisfiability problem. Due to Kamp's Theorem Kam68, we know that $\operatorname{LTL}(\mathrm{U}, \mathrm{S})$ is a fundamental temporal logic since it is as expressive as first-order logic over the class of ordinals. In order to establish this tight complexity characterization, we have introduced the class of simple ordinal automata. This class of automata is more structured than usual ordinal automata and the sets of locations have some structural properties, typically it is a subset of the powerset of some set (herein called the basis). As a consequence, we are also able to improve some results from [Roh97, DN07]. For instance the uniform satisfiability problem is PSPACE-complete and we obtain alternative proofs for results in [DN07]. Recent results about the polynomial space upper bound for LTL over various classes of linear orderings can be found in [Rab10a, Rab10b] by using the so-called composition technique and the automata-based technique used in this paper.

Acknowledgments: We would like to thank the anonymous referees for helpful suggestions and remarks.

\section{REFERENCES}

[BLW07] M. Bezem, T. Langholm, and M. Walicki. Completeness and decidability in sequence logic. In LPAR'07, volume 4790 of Lecture Notes in Computer Science, pages 123-137. Springer, 2007.

[BS73] J.R. Büchi and D. Siefkes. The monadic second order theory of all countable ordinals, volume 328 of Lecture Notes in Mathematics. Springer, 1973.

[Büc62] R. Büchi. On a decision method in restricted second-order arithmetic. In International Congress on Logic, Method and Philosophical Science'60, pages 1-11, 1962.

[Büc64] J.R Büchi. Transfinite automata recursions and weak second order theory of ordinals. In Int. Cong. Logic, Methodology and Philosophy of Science, Jerusalem, pages 3-23, 1964.

[Büc65] J.R. Büchi. Decision methods in the theory of ordinals. Bull. Am. Math. Soc., 71:767-770, 1965.

[Cac06] T. Cachat. Controller synthesis and ordinal automata. In ATVA'06, volume 4218 of Lecture Notes in Computer Science, pages 215-228. Springer, 2006.

[Cri09] J. Cristau. Automata and temporal logic over arbitrary linear time. In FTSETCS'09, volume 4 of Leibniz International Proceedings in Informatics (LIPICs), pages 133-144. LZI, 2009.

[DN07] S. Demri and D. Nowak. Reasoning about transfinite sequences. International Journal of Foundations of Computer Science, 18(1):87-112, 2007.

[DR07] S. Demri and A. Rabinovich. The complexity of temporal logic with until and since over ordinals. In LPAR'0\%, volume 4790 of Lecture Notes in Computer Science, pages 531-545. Springer, 2007.

[GHR94] D. Gabbay, I. Hodkinson, and M. Reynolds. Temporal Logic - Mathematical Foundations and Computational Aspects, Volume 1. OUP, 1994.

[GS85] Y. Gurevich and S. Shelah. The decision problem for branching time logic. JSL, 50(3):668-681, 1985.

[Kam68] J. Kamp. Tense Logic and the theory of linear order. PhD thesis, UCLA, USA, 1968.

[KVW00] O. Kupferman, M. Y. Vardi, and P. Wolper. An automata-theoretic approach to branching-time model checking. Journal of the ACM, 47(2):312-360, 2000.

[Mic84] M. Michel. Algèbre de machines et logique temporelle. In STACS'84, volume 166 of Lecture Notes in Computer Science, pages 287-298. Springer, 1984.

[Rab10a] A. Rabinovich. Temporal logics over linear time domains are in PSPACE, 2010. Submitted to a journal. 
[Rab10b] A. Rabinovich. Temporal Logics over Linear Time Domains are in PSPACE. In RP'10, volume 6227 of Lecture Notes in Computer Science, pages 29-50. Springer, 2010.

[Ram30] F.P. Ramsey. On a problem of formal logic. Proc. London Math. Soc. series 2, 30:264-286, 1930.

[Rey03] M. Reynolds. The complexity of the temporal logic with until over general linear time. Journal of Computer and System Sciences, 66(2):393-426, 2003.

[Rey10a] M. Reynolds. The Complexity of Decision Problems for Linear Temporal Logics. Journal of Studies in Logic, 3(1):19-50, 2010.

[Rey10b] M. Reynolds. The complexity of the temporal logic over the reals. Annals of Pure and Applied Logic, 161(8):1063-1096, 2010.

[Roh97] S. Rohde. Alternating Automata and The Temporal Logic of Ordinals. PhD thesis, University of Illinois, 1997.

[Ros82] J.G. Rosenstein. Linear Orderings. Academic Press, New York, 1982.

[Sav70] W.J. Savitch. Relationships between nondeterministic and deterministic tape complexities. Journal of Computer and System Sciences, 4(2):177-192, 1970.

[SC85] A. Sistla and E. Clarke. The complexity of propositional linear temporal logic. Journal of the ACM, 32(3):733-749, 1985.

[Ven93] Y. Venema. Completeness via completeness: Since and until. In M. de Rijke, editor, Diamonds and Defaults, pages 279-286. Kluwer, 1993.

[VW94] M. Vardi and P. Wolper. Reasoning about infinite computations. Information E Computation, 115:1-37, 1994. 